\title{
Targeting antigen-presenting cells by anti-PD-1 nanoparticles augments antitumor immunity
}

\author{
Farideh Ordikhani, ${ }^{1}$ Mayuko Uehara,, ${ }^{1}$ Vivek Kasinath, ${ }^{1}$ Li Dai, ${ }^{1}$ Siawosh K. Eskandari, ${ }^{1}$ \\ Baharak Bahmani, ${ }^{1}$ Merve Yonar, ${ }^{1}$ Jamil R. Azzi, ${ }^{1}$ Yousef Haik, ${ }^{2}$ Peter T. Sage, ${ }^{1}$ George F. Murphy, ${ }^{3}$ \\ Nasim Annabi, ${ }^{4}$ Tobias Schatton, ${ }^{5}$ Indira Culeria, ${ }^{1}$ and Reza Abdi ${ }^{1}$ \\ 'Transplantation Research Center, Renal Division, Brigham and Women's Hospital, Harvard Medical School, Boston, \\ Massachusetts, USA. ${ }^{2}$ College of Science and Engineering, Hamad Bin Khalifa University, Doha, Qatar. ${ }^{3}$ Department \\ of Pathology, Division of Dermatopathology, Brigham and Women's Hospital, Harvard Medical School, Boston, \\ Masschusetts, USA. ${ }^{4}$ Department of chemical and Biomolecular Engineering, UCLA, California, USA. ${ }^{5}$ Department of \\ Dermatology, Harvard Skin Disease Research Center, Brigham and Women's Hospital, Harvard Medical School, Boston, \\ Massachusetts, USA
}

Recent studies in cancer research have focused intensely on the antineoplastic effects of immune checkpoint inhibitors. While the development of these inhibitors has progressed successfully, strategies to further improve their efficacy and reduce their toxicity are still needed. We hypothesized that the delivery of anti-PD-1 antibody encapsulated in PLCA nanoparticles (anti-PD-1 NPs) to the spleen would improve the antitumor effect of this agent. Unexpectedly, we found that mice treated with a high dose of anti-PD-1 NPs exhibited significantly higher mortality compared with those treated with free anti-PD-1 antibody, due to the overactivation of T cells. Administration of anti-PD-1 NPs to splenectomized LT- $\alpha^{-/-}$mice, which lack both lymph nodes and spleen, resulted in a complete reversal of this increased mortality and revealed the importance of secondary lymphoid tissues in mediating anti-PD-1-associated toxicity. Attenuation of the antiPD-1 NPs dosage prevented toxicity and significantly improved its antitumor effect in the B16-F10 murine melanoma model. Furthermore, we found that anti-PD-1 NPs undergo internalization by $D C s$ in the spleen, leading to their maturation and the subsequent activation of $\mathrm{T}$ cells. Our findings provide important clues that can lead to the development of strategies to enhance the efficacy of immune checkpoint inhibitors.

Authorship note: IG and RA are co-senior authors.

Conflict of interest: The authors have declared that no conflict of interest exists.

License: Copyright 2018, American Society for Clinical Investigation.

Submitted: June 5, 2018 Accepted: September 4, 2018 Published: October 18, 2018

\section{Reference information:} JCI Insight. 2018;3(20):e122700. https://doi.org/10.1172/jci. insight.122700.

\section{Introduction}

Immune responses are initiated in secondary lymphoid tissues (SLT), where naive T cells encounter antigens presented by DCs (1). DCs are conventional antigen-presenting cells (APCs) and play an essential role in the activation and differentiation of T cells (2). The expression of positive costimulatory molecules CD80 and CD86 by DCs is critical to the effective activation of naive T lymphocytes $(1,3)$. Negative costimulatory molecules such as programmed cell death protein 1 (PD-1), on the contrary, limit the activity of proinflammatory $\mathrm{T}$ cells by decreasing their proliferation and ability to produce cytokines, promoting anergy or apoptosis, and potentiating the activity of Tregs (4). The enhancement of the activity of PD-1 has been associated with the attenuation of $\mathrm{T}$ cell-mediated immune diseases (5), and its antagonism has been shown to increase antitumor immunity (6).

Antibody-mediated blockade of PD-1 promotes the conversion of anergic T cells into functional effector T cells, facilitating the antitumor immune response (7-9). Though the development of immune checkpoint inhibitors has advanced the field of immunotherapy significantly, the efficacy of these inhibitors requires improvement. For instance, solitary treatment with the anti-PD-1 mAb nivolumab was found to confer an overall survival rate of only about $50 \%$ over 3 years (10). Furthermore, the onset of toxicity in patients and a steady increase in its incidence are causes for concern (11). Nearly $21 \%$ of patients treated with nivolumab develop treatment-related adverse effects (10). A broad range of treatment-related adverse events occur with PD-1 pathway blockade, including liver and pancreatic toxicity, inflammatory pneumonitis, and interstitial nephritis, all of which may be fatal $(12,13)$. These toxicities are attributable 
to immune overactivation $(14,15)$. Lastly, the high-dose of anti-PD-1 mAb required for a therapeutic outcome substantially increases the cost of treatment (10).

Enhancing the efficacy of checkpoint inhibitors has been the focus of many research efforts, including, for example, use of biomaterial-based delivery systems such as hydrogels (16), microneedle patch-assisted delivery (17), and nanoparticles (NPs) (18-20).

NPs offer numerous advantages, including the improvement in pharmacokinetics and biodistribution of therapeutics, enhancement of tissue penetration and intracellular delivery capacity, and site-specific accumulation of systemically administered therapeutics $(21,22)$. An additional advantage of using NPs is the minimization of the treatment cost, due to the use of a lower dose of anti-PD-1.

A practical target of NP-based drug delivery would be the spleen, considering it is a key SLT populated with APCs that are capable of presenting antigens efficiently to resident $\mathrm{T}$ cells, thereby activating them (23). These T cells can then migrate to areas of injury, including tumor sites $(24,25)$. Due to the action of resident myelosuppressive immune cells, the splenic environment can also produce immune tolerance (26). The spleen, thus, becomes a rational target of which the manipulation could shift the immune response toward a state of activation, thereby enhancing the antitumor efficacy of immune checkpoint inhibitors (27-29). Here, we show that delivery of anti-PD-1 to the spleen via NPs results in a potent antitumor response. Our data show that, despite the use of a low dose of anti-PD-1, delivery of the antibody via NPs results in the maintenance of significant antineoplastic efficacy.

\section{Results}

Characterization of anti-PD-1 NPs. PLGA, a biodegradable and biocompatible synthetic polymer approved for clinical use by the US Food and Drug Administration (FDA), was used to encapsulate anti-PD-1 mAb into NPs (Figure 1A). These anti-PD-1 NPs had a well-defined spherical shape and uniform structure, as depicted in the transmission electron micrograph (Figure 1B). Dynamic light scattering demonstrated that the average hydrodynamic size of anti-PD-1 NPs was about $150 \mathrm{~nm}$ with the polydispersity index (PDI) of about 0.2 (Figure 1C). Release kinetics of anti-PD-1 NPs was determined by incubation at $37^{\circ} \mathrm{C}$ in an orbital incubator shaker and by measuring the released anti-PD-1 mAb by ELISA using recombinant PD-1-coated (rPD-1-coated) plates. Antibody molecules were released from the NPs in a sustained manner over 2 weeks, and their affinity for PD-1 remained preserved throughout this duration (Figure 1D and Supplemental Figure 1A; supplemental material available online with this article; https://doi.org/10.1172/ jci.insight.122700DS1). An encapsulation efficacy of $26.2 \% \pm 3 \%$ was achieved. Low-dose and high-dose anti-PD-1 NPs contained, respectively, $\sim 1.8 \mu \mathrm{g}$ and $\sim 3.6 \mu \mathrm{g}$ anti-PD-1 mAb per $100 \mu 1$ of NPs suspension.

The capacity of anti-PD-1 NPs to promote activation of T cells was evaluated using an allogeneic mixed lymphocyte reaction (MLR) assay. As shown in Figure 1E, anti-PD-1 NP-treated DCs induced proliferation of T cells more robustly than untreated DCs or DCs treated with anti-PD-1 or empty NPs. Flow cytometric analysis demonstrated that this difference in the behavior of anti-PD-1 NP-treated DCs correlated with a higher expression of positive costimulatory molecules CD40, CD80, and CD86 (Figure 1, F-H). DCs could potentially release anti-PD-1 mAb following the uptake of NPs. To assess this possibility, we incubated isolated splenic DCs with APC-labeled anti-PD-1 NPs for 1 hour. Following the incubation, DCs were washed and cultured with T cells for 6 hours. Next, the T cells were analyzed by flow cytometry to assess for the presence of labeled anti-PD-1 mAb in T cells. A high percentage of expression of fluorochrome APC in $\mathrm{CD}^{+} \mathrm{T}$ cells, labeled with the fluorochrome APC, was observed in the group that was coincubated with DCs that were precultured with APC-labeled anti-PD-1 NPs. As expected, the vehicle-treated group did not contain $\mathrm{CD}^{+} \mathrm{T}$ cells that expressed the fluorochrome APC (Supplemental Figure 1B).

Next, we assessed the trafficking of NPs to melanoma tumor by i.v. injecting fluorescently labeled NPs (IR800 NPs) into B16-F10 melanoma tumor-bearing mice followed by live imaging. The NPs immediately distributed throughout the mouse, localized to the tumor at the early time point, and were cleared by 24 hours (Figure 2A). Ex vivo images confirmed that the NPs were not retained indefinitely in the tumor, though they were present in the spleen at 24 hours after injection (Figure 2B). Immunofluorescence staining of splenocytes incubated with NPs for 3 hours showed that the fluorescently labeled NPs were explicitly internalized by CD11 $\mathrm{c}^{+}, \mathrm{F} 4 / 80^{+}$, and $\mathrm{CD} 11 \mathrm{~b}^{+}$cells (Figure $2 \mathrm{C}$ ). Then, we examined the uptake of fluorescently labeled NPs (CF660 NPs) by splenic CD11c $\mathrm{C}^{+}, \mathrm{F} 4 / 80^{+}$, and CD11b cells following i.v. injection. Cytometric analysis of splenocytes 24 hours following injection of NPs showed that NPs were internalized by the CD11 $\mathrm{c}^{+} \mathrm{DCs}, \mathrm{F} 4 / 80^{+}$macrophages, and CD11b $\mathrm{b}^{+}$myeloid cells (Figure 2D). 
A

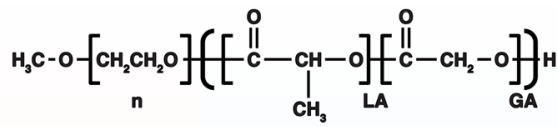

Methoxy Poly(D,L-Lactide-co-Glycolide) Poly(Ethylene Glycol)
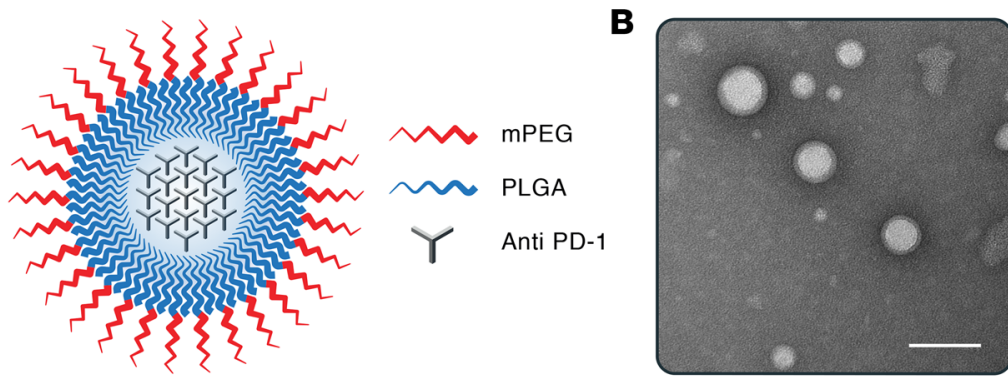

C

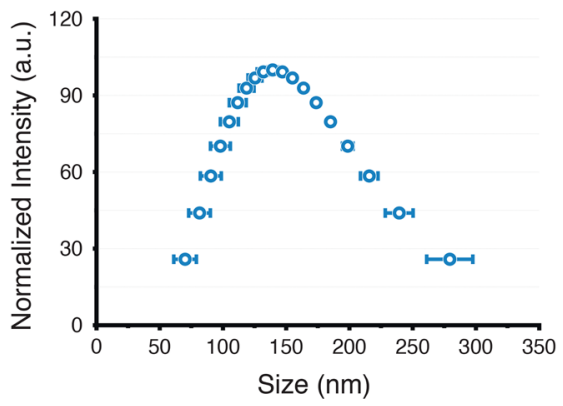

D

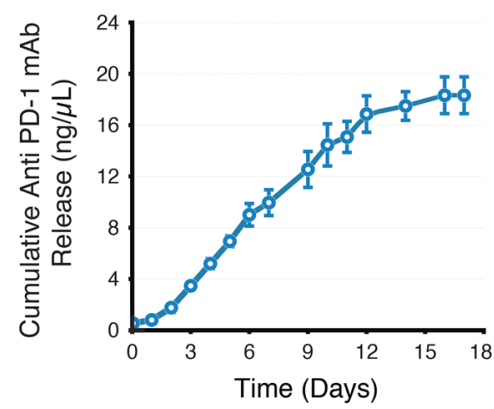

E

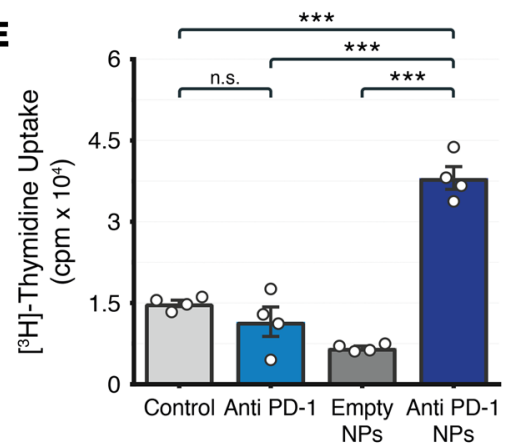

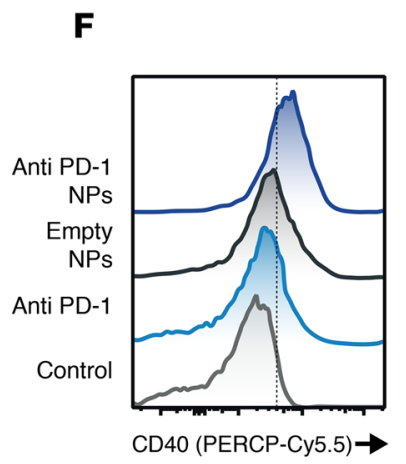

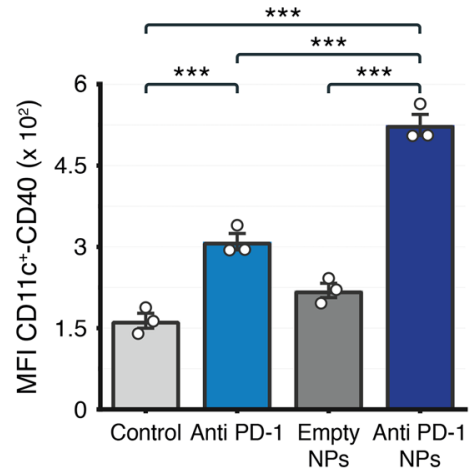

G

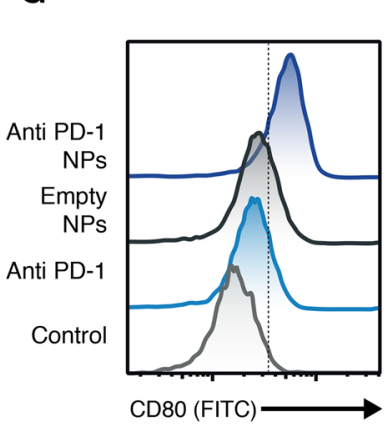

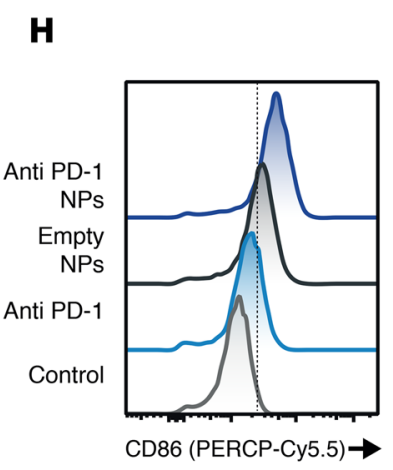

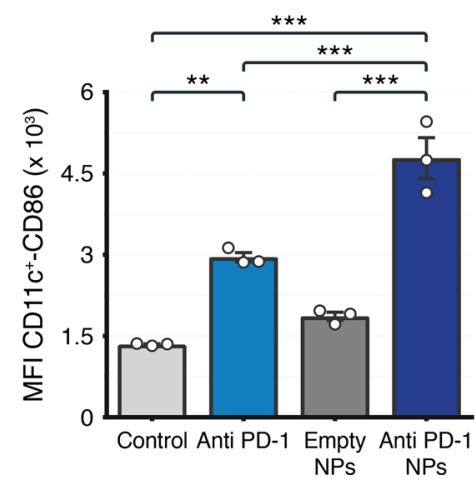

Figure 1. Characterization of anti-PD-1 NPs in vitro. (A) Schematic illustration of anti-PD-1 loaded in PLGA NPs. (B) Representative TEM image of anti-PD-1 NPs, negatively stained, imaged at $80.0 \mathrm{kV}$ (scale bar: $100 \mathrm{~nm}$ ) revealing uniform spherical shape of NPs. (C) Size distribution of antiPD-1 NPs, as measured by DLS, showing the hydrodynamic size of NPs to be $158.3( \pm 4.2) \mathrm{nm}$. (D) Release kinetics of anti-PD-1 mAb from PLGA NPs revealing sustained release of anti-PD-1 mAb over time with activity maintained for 2 weeks. Data are mean \pm SEM of 3 preparations. (E) Anti-PD- 1

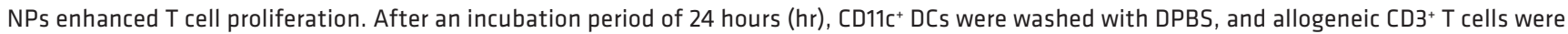
added to these cells. The cells were incubated together for $48 \mathrm{hr}$ at $37^{\circ} \mathrm{C}$ in a humidified atmosphere containing $5 \% \mathrm{CO}_{2}$. The cells were labeled with $\left[{ }^{3} \mathrm{H}\right]$-Thymidine $\left(0.25 \mu \mathrm{Ci}\right.$ per well) for an additional $16 \mathrm{hr}$ to measure T cell proliferation. $\left[{ }^{3} \mathrm{H}\right]$-Thymidine incorporation results show significant proliferation responses to anti-PD-1 NPs in comparison with control, free anti-PD-1 (anti-PD-1), and empty NPs. CPM, counts per minute. (F-H)Representative images and bar graphs show median fluorescence intensities for (F) CD40, (G) CD80, and (H) CD86 among CD11C ${ }^{+}$DCs. Data represent mean \pm SEM. ${ }^{* *} P<0.01 ;{ }^{* *} P<0.005$ calculated by 1 -way ANOVA. 
A
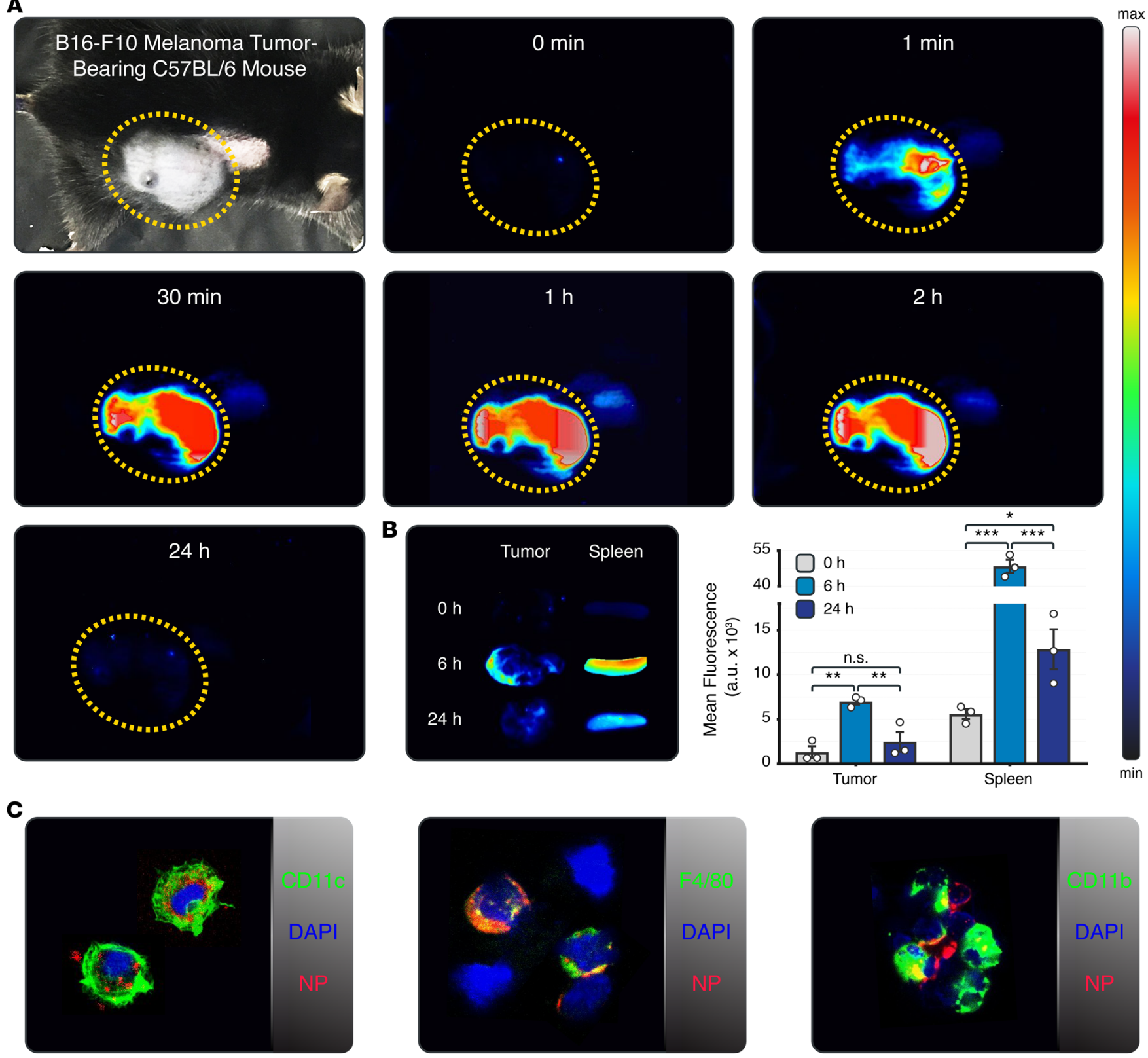

D
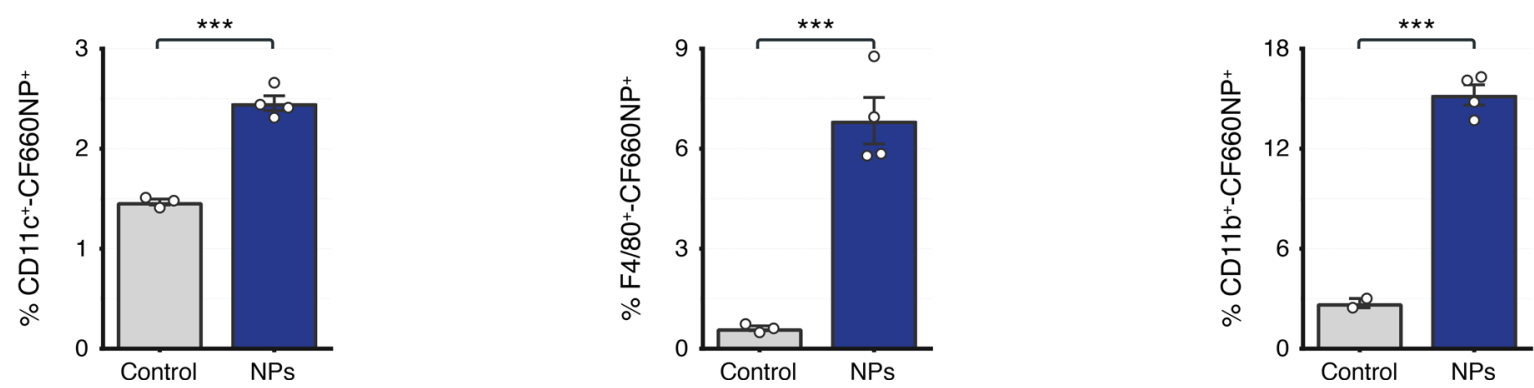

Figure 2. In vitro and in vivo distribution and retention of NPs in melanoma tumor-bearing mice. (A) Representative images of the distribution of IR800 NPs in a melanoma tumor-bearing mouse from 1 minute to $24 \mathrm{hr}$ showing accumulation of NPs at the tumor site. By the 24-hr mark, the NPs are no longer observed. (B) Representative images and quantitative fluorescence intensities of tumors and spleens as calculated from ex vivo images $0 \mathrm{hr}$ (before injection), $6 \mathrm{hr}$, and $24 \mathrm{hr}$ after administration. The data shown are mean \pm SEM ( $n=3-4$ mice/group). (C) Micrograph of splenocytes illustrating localization of NPs within the cytoplasm following $3 \mathrm{hr}$ of incubation in vitro with NPs. Intracellular fluorescence intensities were detected by confocal microscopy. (D) Uptake of CF660 NPs by APCs in the spleens harvested from melanoma tumor-bearing mice 24 hr following injection, as measured by flow cytometry. Data represent mean \pm SEM ( $n=3$ mice/group). Statistical significance was calculated using 1-way ANOVA (B) and 2 -tailed Student's $t$ test (D). ${ }^{*} P<0.05 ;{ }^{* *} P<0.01 ;{ }^{* *} P<0.005$. 
Administration of high-dose anti-PD-1 NPs results in significant T cell activation and death. WT (C57BL/6 WT) mice were treated every other day with anti-PD-1 NPs $(3.6 \mu \mathrm{g}$ anti-PD-1 mAb per $100 \mu \mathrm{l}$ of NPs injection/ mouse) for the lifespan of the study. This group of mice was referred to as the high-dose group, and their overall survival was monitored. Unexpectedly, we found that $~ 70 \%$ of C57BL/6 WT mice ( 5 of 7 treated mice) experienced death within 2 weeks of treatment. On the contrary, mice treated with empty NPs or anti-PD-1 (60 $\mu \mathrm{g}$ per injection/mouse) using the same protocol as above showed no mortality during 1 month of observation.

We hypothesized that immune overactivation played a role in the death of mice treated with high-dose anti-PD-1 NPs. Compared with naive mice and mice treated with anti-PD-1, H\&E-stained lung tissues obtained from mice treated with high-dose anti-PD-1 NPs showed severe interstitial pneumonitis (Figure $3 \mathrm{~A})$. Immunofluorescence staining of the lung additionally revealed extensive infiltration of $\mathrm{CD}^{+} \mathrm{T}$ cells and $\mathrm{CD} 11 \mathrm{~b}^{+}$macrophages (Figure 3B). Moreover, lymphocytic infiltrates were observed in the liver, heart, and pancreas of these mice (Supplemental Figure 2).

We sought to evaluate the functional role of the spleen in the high mortality rate of animals treated with high-dose anti-PD-1 NPs (Figure 3C). Splenectomized mice were treated with high-dose anti-PD-1 NPs as outlined above. Compared with the treated group of WT mice, the splenectomized mice experienced a markedly lower death rate of $25 \%$ ( 1 of 4 treated mice). Considering that lymph nodes could also be sites of immune activation in these mice, we performed the same high-dose treatment regimen in LT- $\alpha^{-1-}$ mice, which notably lack lymph nodes (30). In LT- $\alpha^{-/-}$mice, we also noted a decreased death rate of $25 \%$ ( 1 of 4 treated mice). To address the collective effect of spleen and lymph nodes on the pathogenesis of anti-PD-1 toxicity, we administered a high dose of anti-PD-1 NPs to splenectomized LT- $\alpha^{-/-}$mice. None of these mice died within 30 days of observation ( 5 of 5 treated mice).

Thereafter, we assessed if, indeed, the PD-1 pathway mediated the elevated death rate associated with high-dose anti-PD-1 NPs by treating PD-1 ${ }^{-/-}$mice with anti-PD-1 NPs. No death was observed in this group of mice, either ( 5 of 5 treated mice). These data show that SLTs play a vital role in the pathogenesis of anti-PD-1 NP-mediated organ toxicity and that the death seen in mice receiving treatment is due to engagement of the PD-1 pathway.

Overproduction of cytokines, referred to as cytokine storm, is known to be a key pathologic feature of immune overactivation associated with the use of immunetherapeutics $(31,32)$. To examine the production of inflammatory cytokines in the anti-PD-1 NP-treated mice, we treated the C57BL/6 mice with a high dose of anti-PD-1 NPs every other day for 10 days. Then, the mice were sacrificed 1 hour after the last injection, and their serum was assessed by multiplex assays. Luminex data showed that mice treated with high-dose anti-PD-1 NPs had higher levels of inflammatory Th1 cytokines - specifically IFN- $\gamma$, IL-2, and TNF- $\alpha$ - as compared with the mice in anti-PD-1 mAb- or vehicle-treated groups (Figure 4A). Data for other cytokines are shown in Supplemental Figure 3.

Next, we sought to study the activation status of splenic T cells in mice that received high-dose anti-PD-1 NPs, anti-PD-1, or vehicle. Here, we observed an increase in splenic CD4 ${ }^{+} \mathrm{CD} 69^{+} \mathrm{T}$ cells in mice treated with anti-PD-1 NPs, as compared with the anti-PD-1 mAb- or vehicle-treated mice. Also, the population of effector memory $C D 4^{+} \mathrm{T}$ cells $\left(\mathrm{CD} 44^{\mathrm{hi}} \mathrm{CD} 62 \mathrm{~L}^{\mathrm{lo}}\right)$ was significantly higher in the spleens of the mice treated with anti-PD-1 NPs, as compared with the control groups. No difference in the frequency of Tregs was observed between the 3 groups (Supplemental Figure 4A). Moreover, a higher percentage of $\mathrm{CD}^{+} \mathrm{T}$ cells expressed IFN- $\gamma$ in the spleens of mice receiving anti-PD-1 NPs in comparison with the mice in the vehicle-treated group (Figure 4B). Flow cytometric analysis of splenocyte from C57BL/6 mice indicated that splenocytes from anti-PD-1 NP-treated mice had a significantly higher proportion of $\mathrm{CD} 8^{+} \mathrm{CD} 69^{+}$cells compared with the splenocytes from vehicle-treated mice. No difference was observed in the proportion of $\mathrm{CD} 8{ }^{+} \mathrm{CD} 44^{\text {hi }} \mathrm{CD} 62 \mathrm{~L}^{\text {lo }}$ effector memory and $\mathrm{CD} 8^{+} \mathrm{IFN}-\gamma^{+} \mathrm{T}$ cells in the splenocytes from anti-PD-1 NP-treated mice compared with anti-PD-1 and vehicle-treated mice (Supplemental Figure 4B).

Anti-PD-1 NPs improve the efficacy of anti-PD-1 in suppression of murine melanoma tumor. To characterize and study the functionality and efficacy of anti-PD-1 NPs in the treatment of melanoma, we used the B16-F10 mouse model. Notably, to minimize the side effects of anti-PD-1 NPs in the B16-F10 melanoma tumor-bearing mice, we decreased the dosage ( $1.8 \mu \mathrm{g}$ anti-PD-1 mAb per $100 \mu \mathrm{l}$ of NPs injection/mouse) and the frequency of injections $(2 \times$ week). Lowering the cumulative dosage of anti-PD-1 NPs prevented toxicity, as demonstrated by the lack of difference in the number of infiltrating lymphocytes in the tissues of mice treated with anti-PD-1 NPs, as compared with those of mice treated with anti-PD-1 or vehicle (Supplemental Figure 5). 
A

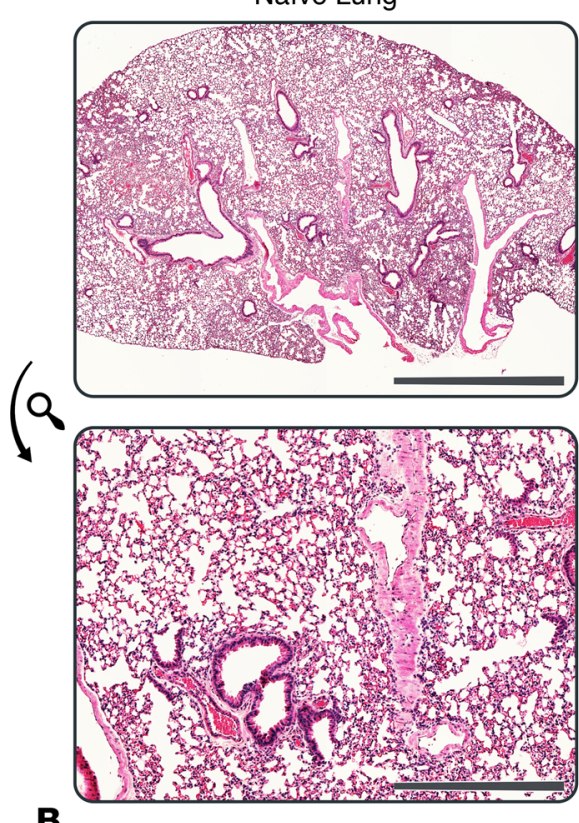

B

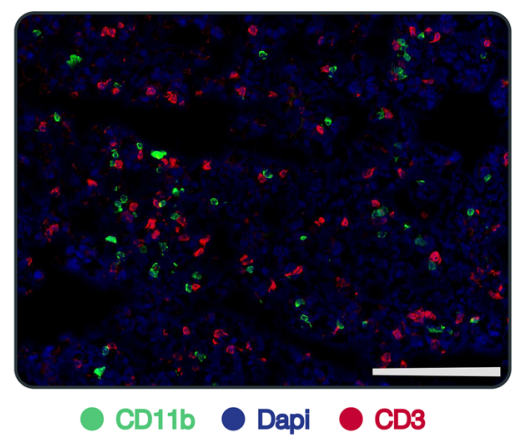

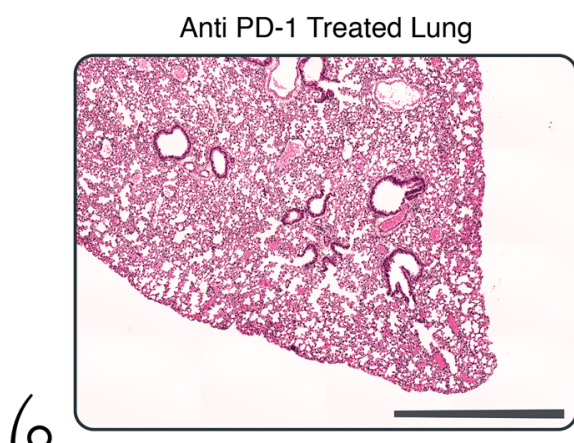

Q
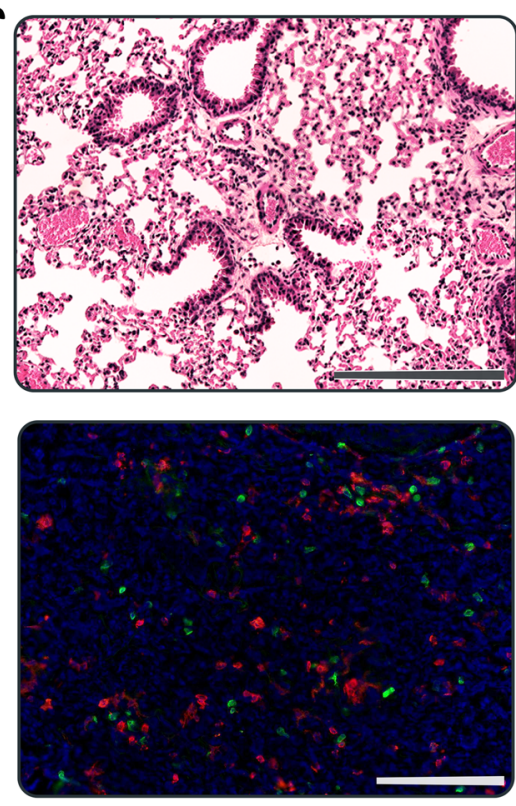

CD11b

Dapi

CD3

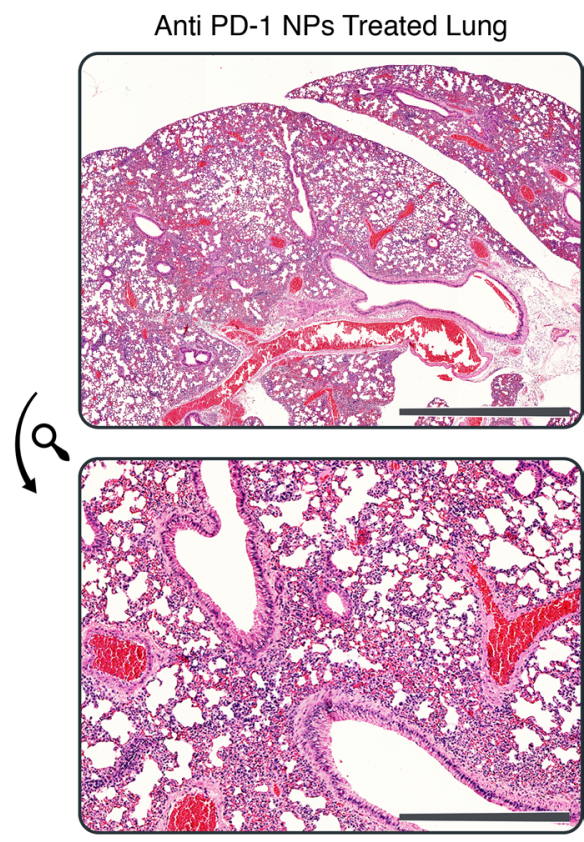

\section{C}

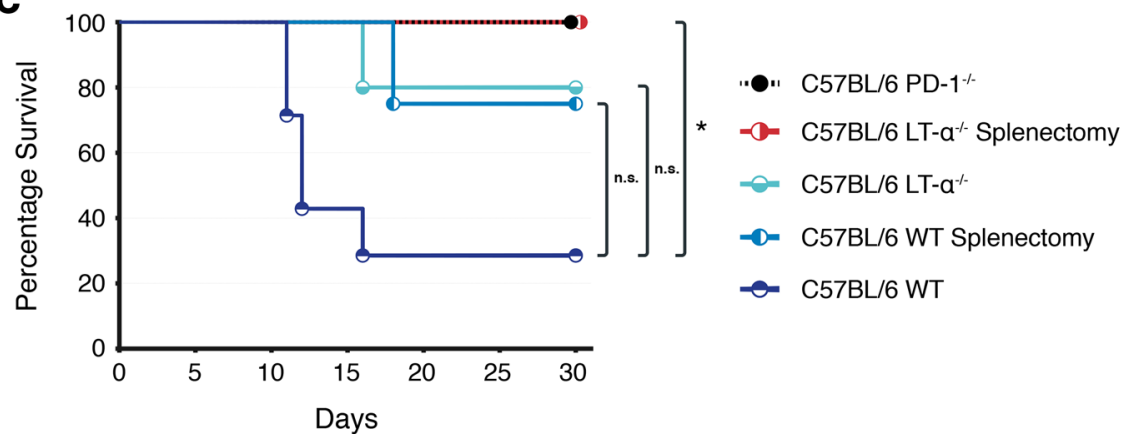

Figure 3. Administration of high-dose anti-PD-1 NPs results in significant T cell activation and death. (A) Light micrograph of H\&E-stained lung tissue from anti-PD-1 NP-treated mice reveals higher cellular infiltration, indicative of interstitial pneumonitis, as compared with the lungs from antiPD-1-treated and naive mice. These images are representative of 2 sections of 3-4 different mice in each group. (B) Immunofluorescence micrograph of lung tissue showing extensive infiltration of $\mathrm{CD}^{+} \mathrm{T}$ cells and CD11 b+ macrophages. Scale bars: (from top to bottom) $2,000 \mu \mathrm{m}, 500 \mu \mathrm{m}$, and $100 \mu \mathrm{m}$. These images are representative of 3 sections of 3-4 different mice in each group. (C) Kaplan-Meier survival curves for the C57BL/6 and LT- $\alpha^{-/-}$mice with and without splenectomy, as well as PD-1/- mice following high-dose anti-PD-1 NPs treatment every other day for the lifespan of the study (3.6 $\mu \mathrm{g}$ anti-PD-1 mAb per $100 \mu \mathrm{l}$ of NPs injection/mouse). C57BL/6 WT mice had a higher rate of mortality compared with splenectomized C57BL/6 mice, LT- $\alpha^{-1-}$ mice, and PD-1-1- mice ( $n=4-7$ mice/group). ${ }^{*} P<0.05$, calculated by the log-rank test (Mantel-Cox).

To study whether prophylactic treatment of melanoma with anti-PD-1 NPs resulted in a decrease in tumor growth, we treated mice 1 day prior to inoculation of melanoma cells. As shown in Figure 5A, treatment with anti-PD-1 NPs resulted in a reduction in tumor size, as compared with treatment with an equivalent dose of anti-PD-1 mAb, empty NPs, or vehicle. The average tumor size for vehicle-, empty NP-, anti-PD-1-, and 
A

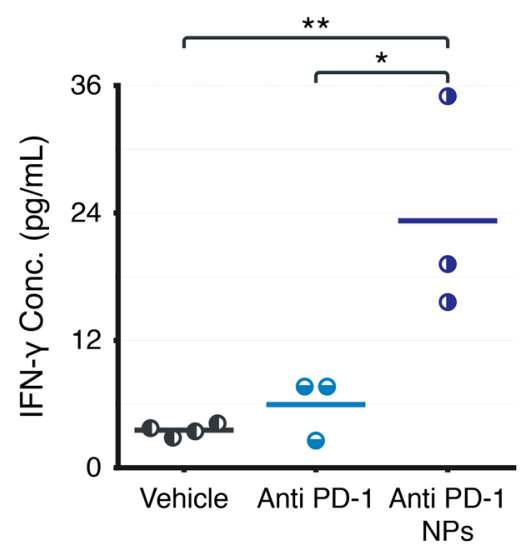

B

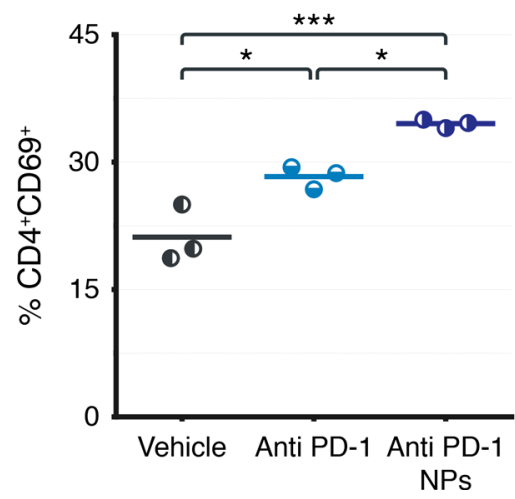

Vehicle Ant PD-1 $\bigcirc$ Antl PD-1 NPs
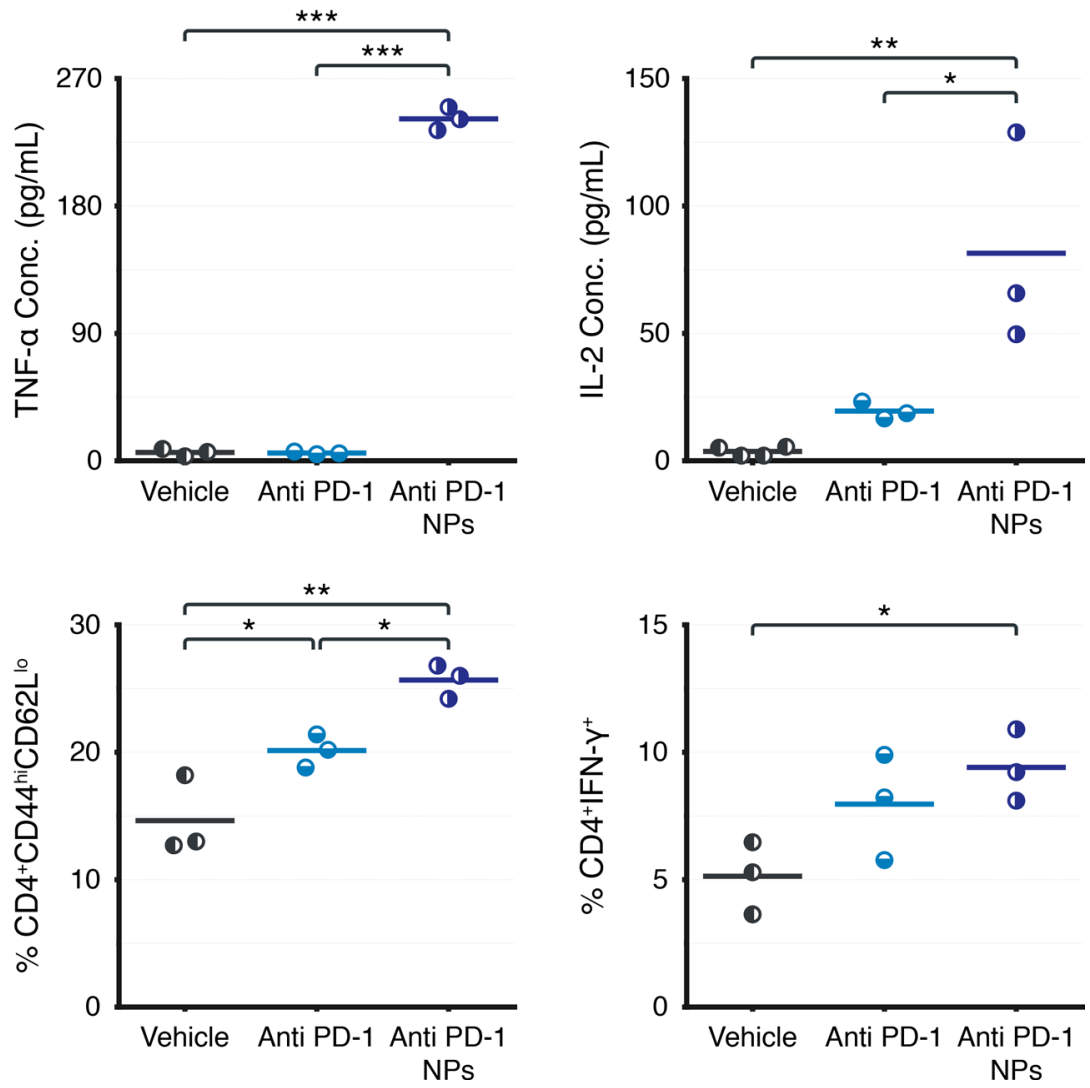

Figure 4. Cytokine profile and T cell immune activation status of mice following treatment with high-dose anti-PD-1 NPs. Mice received anti-PD-1 NPs every other day for 10 days. (A) Luminex analysis indicating a significant increase in Th1 cytokine levels in plasma of mice treated with anti-PD-1 NPs. (B) Flow cytometric analysis from C57BL/6 mice indicating that the splenocytes from anti-PD-1 NP-treated mice had a significantly higher proportion of CD4+CD69+, CD4 ${ }^{+}$CD44 ${ }^{\text {hi }} \mathrm{CD}_{22} \mathrm{~L}^{10}$ effector memory, and CD4 ${ }^{+} \mathrm{FN}-\gamma^{+} \mathrm{T}$ cells, as compared with free anti-PD-1 (anti-PD-1) and vehicle-treated mice. The data are representative of 2 independent experiments. Data represent mean \pm SEM $\left(n=3-4\right.$ mice/group). ${ }^{*} P<0.05 ;{ }^{* *} P<0.01 ;{ }^{* *} P<0.005$, calculated by 1-way ANOVA.

anti-PD-1 NP-treated mice were $915( \pm 397), 814( \pm 553), 1,111( \pm 961)$, and $322( \pm 289) \mathrm{mm}^{3}$ respectively, 17 days following tumor inoculation ( $n=5$ mice/group).

Next, we assessed the efficacy of the anti-PD-1 NPs in the treatment of established tumors. Mice were implanted with melanoma tumor cells, and the tumor size was allowed to reach to $25-30 \mathrm{~mm}^{3}$. Then, the mice were randomized into different groups and treatment began with injecting of different therapeutics. Twenty-four days following tumor inoculation, the average tumor size for the vehicle-, empty NP-, anti-PD-1-, and anti-PD-1 NP-treated mice were 1,242 ( \pm 133), 1,385 ( \pm 388), $802( \pm 348)$, and $580( \pm$ 208) $\mathrm{mm}^{3}$, respectively, ( $n=6-7$ mice/group). Treatment with anti-PD-1 NPs decelerated tumor growth significantly in comparison with treatment with empty NPs or vehicle. Though there was a trend toward improved efficacy, no statistical difference was found between the tumor size of anti-PD-1- and anti-PD-1 NP-treated mice (Figure 5B). Additionally, the mean tumor growth inhibition percentage, measured 24 days following the inoculation of melanoma, was higher in the mice that received anti-PD-1 NPs (53.24\%), as compared with the mice that received the same dosage of anti-PD-1 (35.42\%). Linear regression was used to compare the slopes of the 2 groups, which revealed average tumor growth slopes $34 \pm 5.5$ and $23 \pm$ 4 for mice treated with anti-PD-1 and anti-PD-1 NPs, respectively $(P<0.01)$.

The potential mechanisms by which anti-PD-1 NPs evoke potent antitumor effects were also studied. Melanoma tumor-bearing mice treated with anti-PD-1 NPs, anti-PD-1, or vehicle were sacrificed 17 days after tumor inoculation. Splenocytes were subjected to flow cytometry to assess the relative abundance of activated $\mathrm{T}$ cells in the different groups. Anti-PD-1 NP-treated mice exhibited significant increases in the percentages of effector splenic $\mathrm{CD} 4^{+} \mathrm{CD} 44^{\mathrm{hi}} \mathrm{CD} 62 \mathrm{~L}^{\text {lo }}$ and $\mathrm{CD} 8^{+} \mathrm{CD} 44^{\mathrm{hi}} \mathrm{CD} 62 \mathrm{~L}^{\text {lo }} \mathrm{T}$ cells compared with mice treated with anti-PD-1 or vehicle (Figure 6A). Moreover, significantly higher proportions of both $\mathrm{CD} 4^{+}$and $\mathrm{CD} 8^{+} \mathrm{T}$ cells in 
A

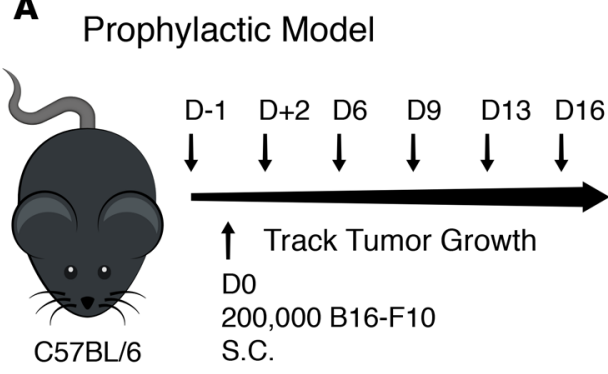

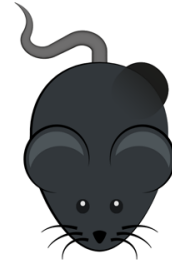

C57BL/6

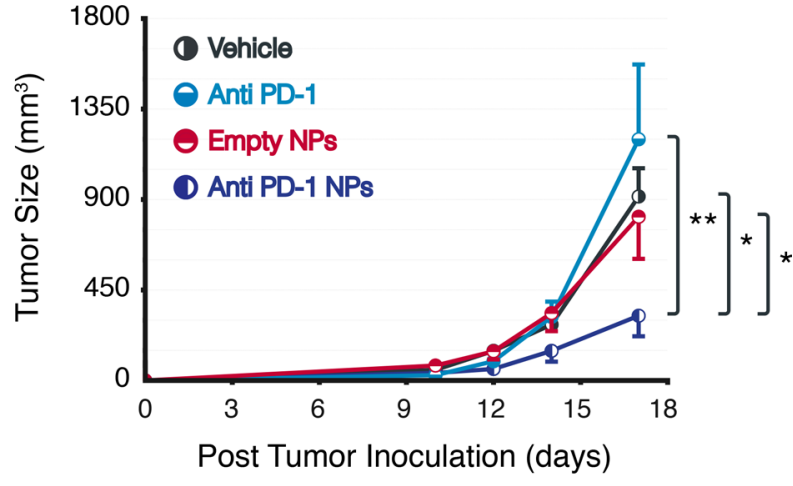

B

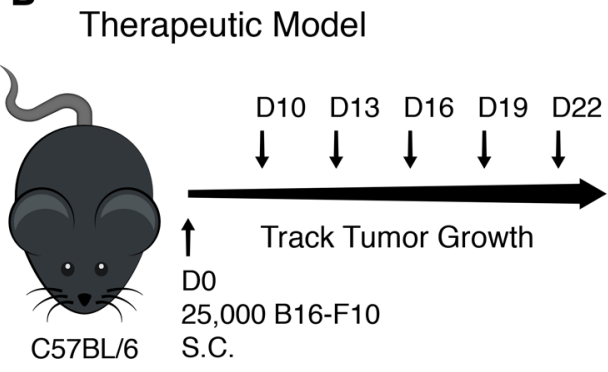

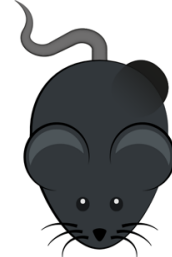

C57BL/6

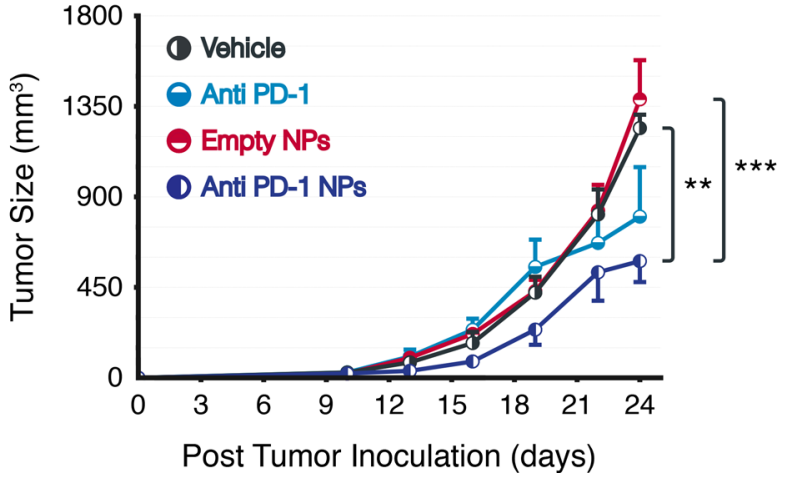

Figure 5. Anti-PD-1 NPs decreases tumor growth in a B16-F10 murine melanoma model. (A) In vivo treatment plan and tumor growth kinetics in a prophylactic model. C57BL/6 mice received the treatment 1 day prior to inoculation of B16-F10 melanoma cells, and the sizes of the tumors were compared at day 17 with Student's $t$ test ( $n=5$ mice/group). Data represent mean \pm SEM. (B) In vivo treatment plan and tumor growth kinetics in a therapeutic model. Treatment started at 10 days after B16-F10 melanoma cell inoculation in C57BL/6 mice ( $n=6-7$ mice/group), and the sizes of the tumors were compared at day 24 with Student's $t$ test. Data represent mean \pm SEM. ${ }^{*} P<0.05$; ${ }^{*} P<0.01 ;{ }^{* *} P<0.005$, calculated by $t$ test.

the spleens of mice treated with anti-PD-1 NPs had an activated $\mathrm{CD} 69^{+}$phenotype, compared with the $\mathrm{CD} 4^{+}$ and $\mathrm{CD} 8^{+} \mathrm{T}$ cells in mice treated with anti-PD-1 or vehicle (Figure 6B). Given that IFN- $\gamma$ is a critical purveyor of antitumor immunity, we examined the expression of IFN- $\gamma$ by splenocytes, as well. Higher percentages of $\mathrm{CD} 4^{+} \mathrm{T}$ cells in the spleen of mice treated with anti-PD-1 NPs expressed the Th1 cytokine IFN- $\gamma$, as compared with those from mice that received anti-PD-1 or vehicle (Figure 6C). Treatment with anti-PD-1 NPs, however, did not significantly alter the percentage of $\mathrm{CD} 8^{+} \mathrm{T}$ cells expressing IFN- $\gamma$ in the spleen.

Additionally, a higher percentage of $\mathrm{CD} 11 \mathrm{c}^{+} \mathrm{CD} 86^{+} \mathrm{DC}$ s was observed in the spleens of anti-PD-1 $\mathrm{NP}-$ treated mice, as compared with those from the mice that received anti-PD-1 or vehicle (Figure 6D). This finding corroborates our results discussed previously, which demonstrated that in vitro treatment with anti-PD-1 NPs resulted in the activation of CD11 $\mathrm{c}^{+}$DCs (Figure 1, F-H).

We also investigated whether anti-PD-1 NPs affects the phenotype of $\mathrm{T}$ cells in melanoma tumors. Melanoma tumors in mice treated with anti-PD-1 NPs contained a higher percentage of $\mathrm{CD} 4^{+}$and $\mathrm{CD} 8^{+}$ $\mathrm{T}$ cells, compared with the tumors in mice that received anti-PD-1 or vehicle (Figure 7, A and B). As shown in Figure $7 \mathrm{C}$, the ratio between $\mathrm{CD} 8^{+} \mathrm{T}$ cells and $\mathrm{CD} 4^{+} \mathrm{CD} 25^{+} \mathrm{FOXP} 3^{+}$Tregs $\left(\mathrm{CD} 8^{+} / \mathrm{CD} 4^{+} \mathrm{CD} 25^{+}\right.$ $\mathrm{FOXP}^{+}$), which reflects the therapeutic efficacy of tumor immunotherapy, was 4- to 5-fold higher in mice that received anti-PD-1 NPs versus anti-PD-1 or vehicle.

\section{Discussion}

Immune checkpoint blockade with anti-PD-1 agents has demonstrated impressive benefits in the treatment of several cancers (33). However, the toxicity associated with immune checkpoint inhibitors, such as antiPD-1/PD-L1 therapeutics, has grown to be a significant medical problem $(7,34)$. These complications, such as pneumonitis, have been described in nearly $10 \%$ of patients who receive anti-PD-1/PD-L1 therapy, 
A

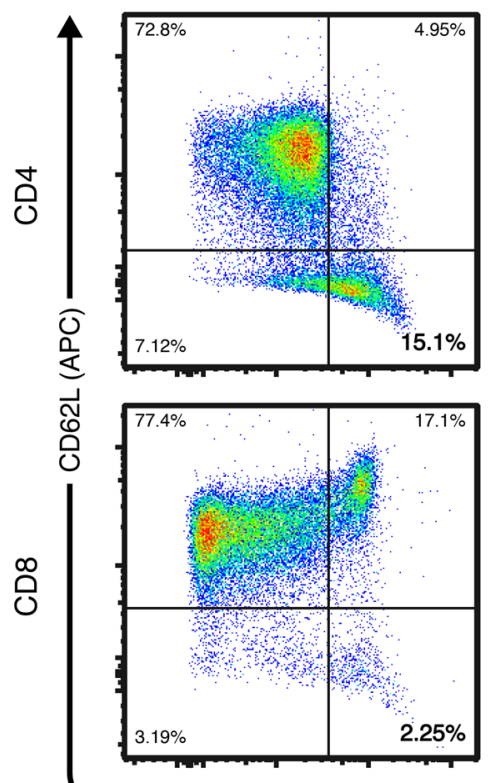

B

B

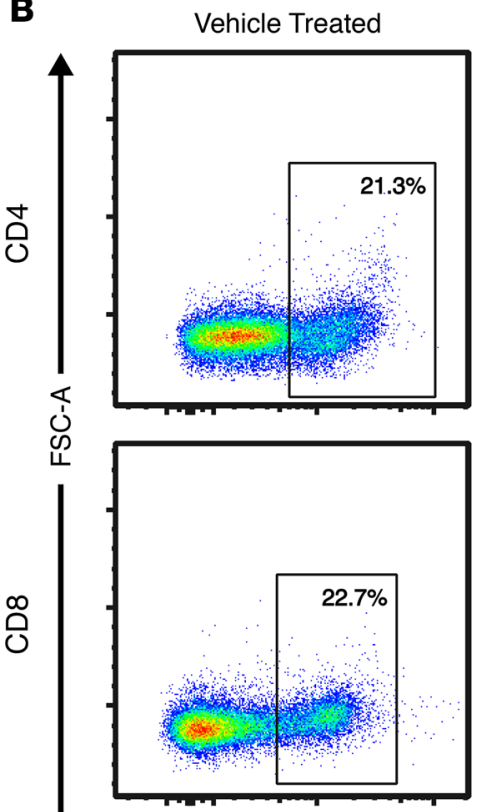

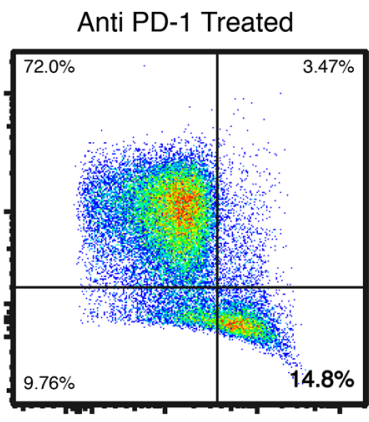

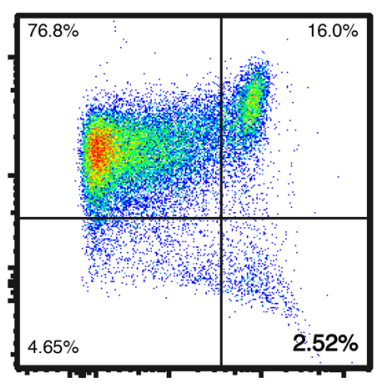

CD44 (PERCP-Cy5.5)

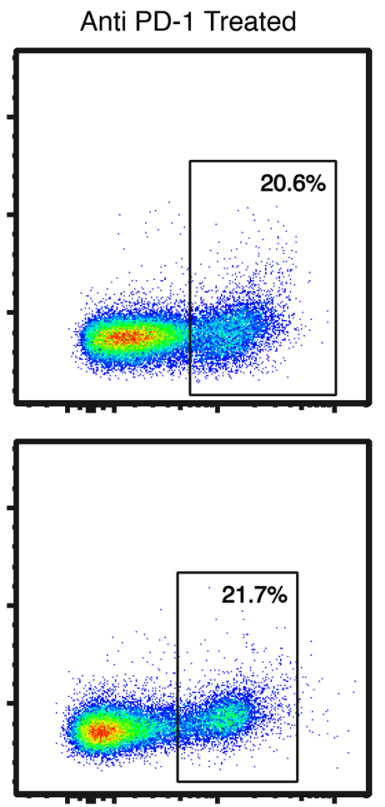

CD69 (PE-Cy7)
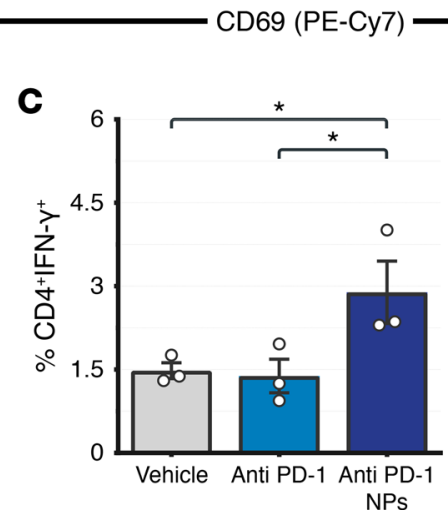

Anti PD-1 NPs Treated
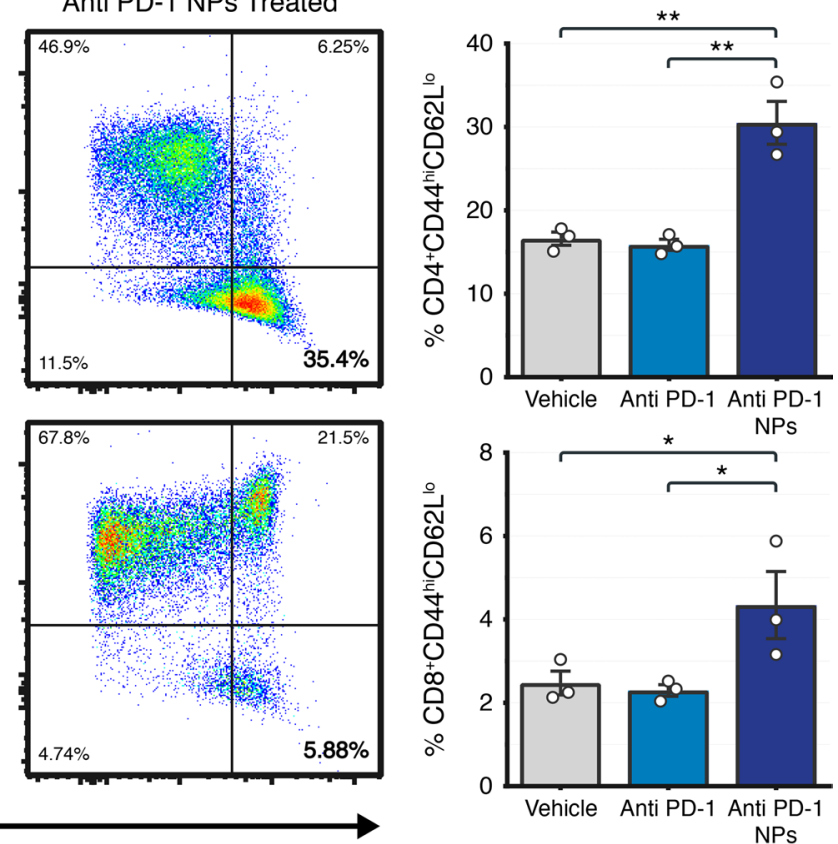

NPs
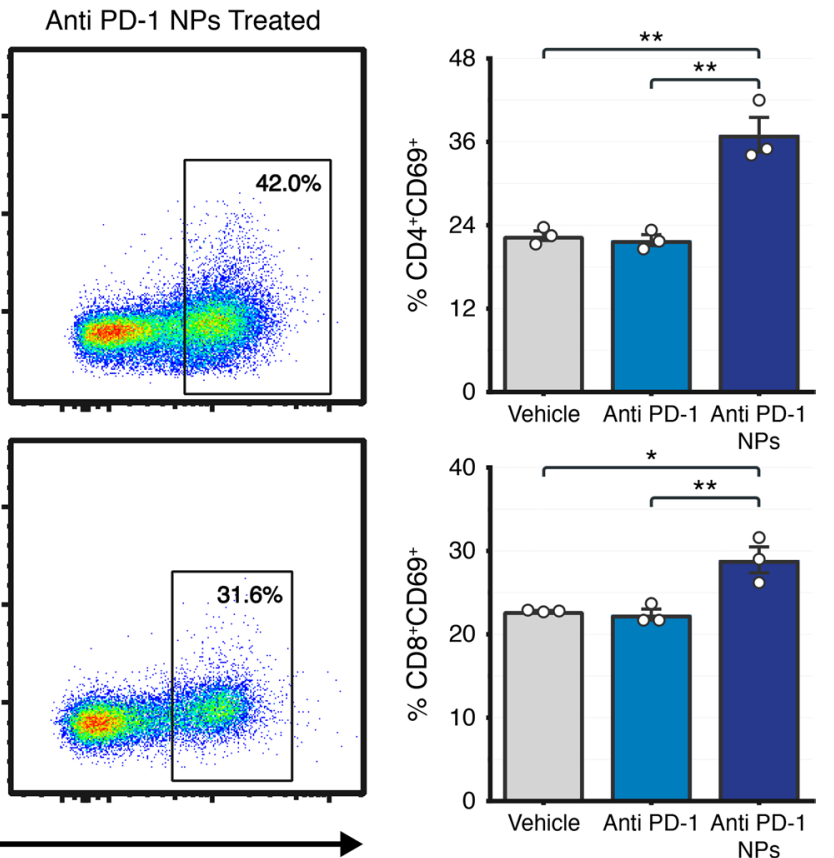

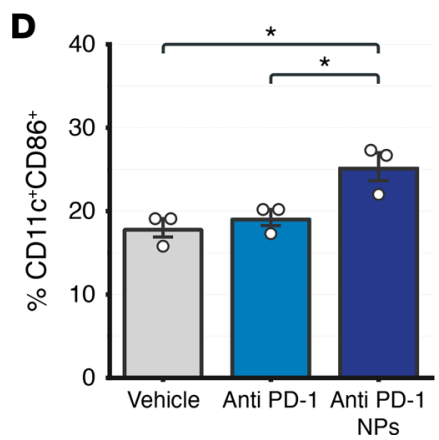


Figure 6. T cell profile of the spleens from anti-PD-1 NP-treated B16-F10 melanoma tumor-bearing C57BL/6 mice at day 17 following tumor cell inoculation. (A and B) The spleens of mice in the anti-PD-1 NP-treated group had higher percentages of $C D 4^{+}$and $C D 8^{+}$effector memory T cells, CD4+CD69+ $T$ cells, and CD8 ${ }^{+} \mathrm{CD}_{69}{ }^{+} \mathrm{T}$ cells in comparison with anti-PD-1- or vehicle-treated mice. (C) The percentage of CD4+IFN- $\gamma^{+} \mathrm{T}$ cells was significantly higher in the spleens of mice treated with anti-PD-1 NPs than in those of mice receiving free anti-PD-1 (anti-PD-1) or vehicle. (D) A significantly higher percentage of CD11c ${ }^{+}$CD86 ${ }^{+}$cells was observed in the spleens of mice treated with anti-PD-1 NPs in comparison with those of mice treated with free anti-PD-1 or vehicle. Data are presented as mean \pm SEM $\left(n=3\right.$ mice/group). ${ }^{*} P<0.05$; ${ }^{*} P<0.01$, calculated by 1 -way ANOVA.

either alone or in combination with other drugs (7). Moreover, in addition to being frequent, these complications can be lethal.

The recent advent of engineered $\mathrm{mAb}$ has significantly changed the course of refractory diseases. However, several limitations impede their full utility. For instance, large antibodies might have poor tissue penetration. Strategies using $\mathrm{mAb}$ often rely on several injections of $\mathrm{mAb}$, which could increase the cost and may raise issues with patient compliance. Nanotechnology improves not only the pharmacokinetics of the therapeutic payload, but it also allows for site-specific targeted drug delivery (35-37). Using NPs can enhance tissue penetration and the capacity for intracellular delivery of antibodies. The NPs that have been designed herein have strong potential for clinical translation, as they are prepared from FDA-approved PEG and PLGA polymers. Physical characterization of our NPs demonstrated a spherical morphology with an average size of $\sim 150 \mathrm{~nm}$ and an encapsulation efficacy of $26.2 \% \pm 3 \%$. In vitro release kinetics of anti-PD-1 mAb from the NPs revealed sustained release of anti-PD-1 over time. Using centrifugal filter units significantly reduced the nonspecific binding of the antibody to the surface. Hence, this method markedly reduced the burst release and allowed for controlled, sustained release of anti-PD-1 mAb over time.

As SLTs are the primary sites for immuneactivation, circulating immune cells migrate to the spleen, where they can encounter antigens presented by APCs, such as DCs. Following activation, T cells infiltrate the tumor site and can effectively kill tumor cells. Our goal was to develop a simple and clinically feasible approach that would allow delivery of anti-PD-1 to the SLTs.

The spleen is a major SLT particularly well perfused by blood $(\sim 350 \mathrm{ml} / \mathrm{min})$, which facilitates the delivery of the payload. Importantly, the marginal zone that surrounds the white pulp region of the spleen contains numerous APCs, including DCs. As such, the immune response against tumor antigens in the spleen can be enhanced by tipping the homeostasis either toward immunoregulatory and suppressive cells or immunogenic effector cells. Of note, in addition to immune activation, peripheral tolerance toward antigens (including tumor antigens) can originate in and develop from the spleen $(23,38,39)$.

Previous studies have established the propensity of NPs to become sequestered in the spleen; however, to improve the efficacy of targeted delivery of NPs, meaningful efforts are underway to reduce splenic trafficking $(40,41)$. In this study, on the contrary, we hypothesized that optimization of the payload of anti-PD-1 delivered to the DCs of the spleen would increase its capacity to suppress tumor growth. Martin et al. were the first to show that allogeneic splenocytes cross-linked with ethylene carbodiimide induced splenic tolerance (42). Leuschner et al. have shown that delivery of siRNA that targets CCR2-expressing monocytes could abrogate inflammatory responses in atherosclerotic plaques (41). Others have also demonstrated that PLGA NPs deliver tumor-specific antigens to DCs in a protected and sustained-release manner (43-45). In this study, we demonstrated that the delivery of anti-PD-1 within PLGA NPs facilitated its uptake by DCs and enhanced the effector T cell response in the SLTs.

In our study, WT mice treated with high-dose anti-PD-1 NPs exhibited a high rate of mortality. Histopathological analysis of organs from these mice revealed massive infiltration of immune cells, which was especially prominent in the lungs and corroborated the reports of severe toxic effects of anti-PD-1 $\mathrm{mAb}$ drugs (46-48). Removal of the spleen reduced the rate of death in these animals significantly. As the lymph node is also a major component of SLTs, we additionally showed that LT- $\alpha^{-/-}$mice, in which lymph nodes are absent, were also protected against death. Notably, removal of the spleen in LT- $\alpha^{-/}$ mice completely protected these mice from death. The observation that $\mathrm{PD}-1^{-1-}$ mice were also protected (Figure 3C) implies that the blockade of the PD-1 signaling pathway is similarly essential for the protection from death. Results provided here have significant clinical implications, given the dramatic increase in the use of checkpoint inhibitors and the rise in the incidence of toxicities associated with their use.

In line with the above observation, to decrease the toxicity of anti-PD-1 NPs, we were able to lower the concentration of anti-PD-1 and decrease the frequency of injections. No noticeable difference in infiltrating lymphocytes was observed in the nonlymphoid tissues of mice that received a low dose of anti-PD-1 NPs, compared with the mice that received vehicle or anti-PD-1. 
A

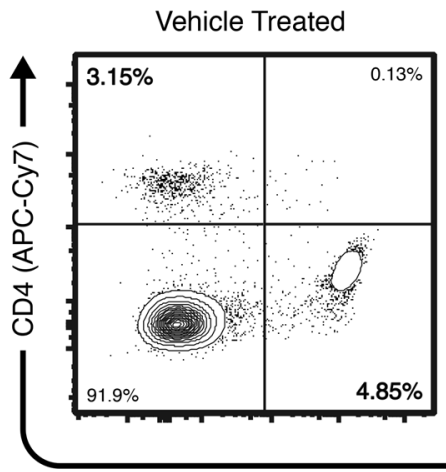

B

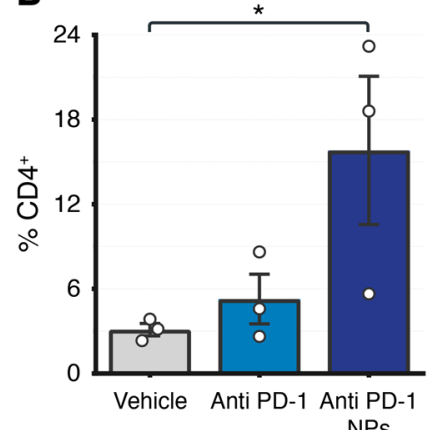

Anti PD-1 Treated

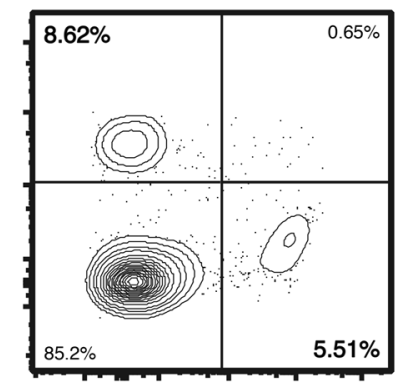

CD8 (PERCP-Cy5.5)

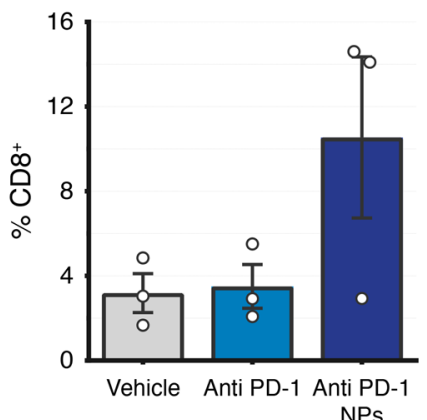

Anti PD-1 NPs Treated

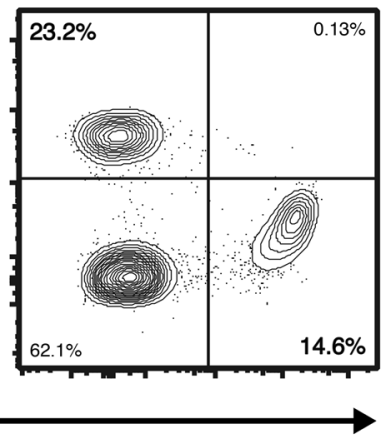

C

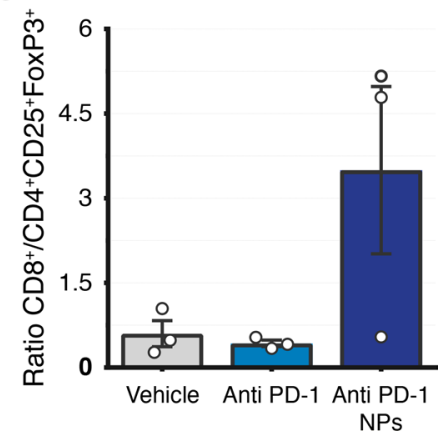

Figure 7. T cell profile of the tumors from anti-PD-1 NP-treated B16-F10 melanoma tumor-bearing C57BL/6 mice at day 17 following tumor cell inoculation. (A and $\mathbf{B}$ ) Analysis of $C D 4^{+} T$ cells and $C D 8^{+} T$ cells showing highest percentages of these cells in the melanoma tumors of mice treated with anti-PD- 1 NPs. (C) The ratio of $C D 8^{+} / C D 4^{+} C D 25^{+} F O X P 3^{+}$in the melanoma tumors was higher in mice that received anti-PD-1 NPs versus free anti-PD-1 (anti-PD-1) or vehicle. Data are presented as mean \pm SEM ( $n=3$ mice/group). ${ }^{*} P<0.05$; calculated by 1 -way ANOVA.

Furthermore, we evaluated the effect of anti-PD-1 NPs in B16-F10 mice, a highly stringent animal model of melanoma. Our data exhibited that treatment with anti-PD-1 NPs in both the prophylactic and therapeutic treatment modalities demonstrated efficacy in reducing tumor growth rate. The cumulative dosage of anti-PD-1 administered via our NP $(10.8 \mu \mathrm{g}$ and $9 \mu \mathrm{g}$ for the prophylactic and therapeutic modalities, respectively) was significantly lower than the amount of anti-PD-1 $(2,200 \mu \mathrm{g})$ used in a previous experiment, which showed no therapeutic effect (49).

Mechanistically, in addition to the general activation of $\mathrm{T}$ cells, the shifting of differentiated $\mathrm{T}$ cells toward a $\mathrm{CD} 4^{+} \mathrm{Th} 1$ cell phenotype has been shown to protect against tumors through the production of IFN- $\gamma$. Consistent with this mechanism, we observed a high number of IFN- $\gamma$-producing CD $4^{+} \mathrm{T}$ cells in the spleens of the anti-PD-1 NP-treated mice.

In addition to its cytotoxic effect, IFN- $\gamma$ has recently been shown to induce fragility of Tregs (50). Whether IFN- $\gamma$ affected the fragility of Tregs in our model remains to be studied. Although IFN- $\gamma$ has been shown to promote tumor development in certain tumor models $(51,52)$, our data suggest that IFN- $\gamma$ can modulate the immune response in favor of an antitumor effect. Furthermore, the proportion of effector memory $\mathrm{CD} 8^{+} \mathrm{T}$ cells in the spleen of mice receiving anti-PD-1 NPs was increased, and these cells were highly activated $\left(\mathrm{CD} 8^{+} \mathrm{CD} 69^{+}\right)$, which likely contributed to the enhanced clearance of the tumor in our model. The activation of cytotoxic $\mathrm{T}$ cells $\left(\mathrm{CD} 8^{+} \mathrm{CD} 69^{+}\right)$by treatment with anti-PD-1 NPs can also be attributed to IFN- $\gamma$ signaling, as another tumor model has demonstrated (53). While we show that absence of lymph nodes reduces the mortality rate, future studies are required to assess the microenvironment of the draining lymph nodes for immune activation and drug delivery (54). Interestingly, the local populations of $\mathrm{CD}^{+}$and $\mathrm{CD} 8^{+} \mathrm{T}$ cells at the tumor site were increased in the mice that received anti-PD-1 NPs, but the activation status of these cells did not change. We observed a trend toward an increased ratio of $\mathrm{CD} 8^{+} \mathrm{T}$ cells to $\mathrm{CD} 4^{+} \mathrm{CD} 25^{+} \mathrm{FOXP}^{+}$Tregs in the tumor following treatment with anti-PD-1 NPs. This ratio determines the balance between the proinflammatory $\left(\mathrm{CD} 8^{+}\right)$versus antiinflammatory $\left(\mathrm{CD} 4^{+} \mathrm{CD} 25^{+} \mathrm{FOXP} 3^{+}\right)$response against the tumor. The increased ratio observed in our study suggests a more dominant role of $\mathrm{CD} 8^{+} \mathrm{T}$ cells 
in enhancing the clearance of the tumor. A higher ratio of $\mathrm{CD} 8^{+}$to $\mathrm{CD} 4^{+} \mathrm{CD} 25^{+} \mathrm{FOXP} 3^{+} \mathrm{T}$ cells has also been shown to be a prognostic marker for the survival of cancer patients (55). The finding that our NPs traffic to the tumor following systemic delivery suggests that the delivery of anti-PD-1 directly to the tumor may also have resulted in an intensification of local antitumor immunity (56). The lack of discernable impacts on tumor-infiltrating immune cells could be due to the timing at which we investigated the tumor microenvironment. Additionally, much of the antitumor effects could be due to soluble mediators or cytokines that are more complicated to assess than the mere enumeration of immune cells because of the kinetics and short stability of some of the cytokines - specifically, cytokines such as IL-2 and TNF- $\alpha$ are difficult to measure because of their labile nature.

Tumor-derived factors suppress the full maturation of DCs, thereby affecting their ability to process and present antigens (3). In contrast, mature DCs can activate $\mathrm{CD}^{+}$and $\mathrm{CD} 8^{+}$effector memory $\mathrm{T}$ cells in the spleen, which results in enhanced antineoplastic activities (57). APCs induce antitumor immune responses via efficient processing and presentation of tumor antigens, followed by subsequent priming of tumor antigen-specific $\mathrm{T}$ cells. These effector $\mathrm{T}$ cells participate in the eradication of the tumor at the tumor site (58). We observed high expression of the positive costimulatory molecule CD86 on the DCs in the spleens of anti-PD-1 NP-treated mice, suggesting an improvement in the maturation and activation of these cells. CD86 ${ }^{+}$DCs can present tumor antigens more effectively to prime antitumor cytotoxic and helper T cells, as has been reported earlier (59).

Failure of any of these steps suppresses antitumor immune responses, and therefore the interest in understanding these processes both under normal and pathophysiological conditions has intensified.

Collectively, our study shows that the administration of anti-PD-1 NPs can lead to antitumor immunity in the murine B16-F10 melanoma model. Potential mechanisms for this superior antitumor immune response mediated by anti-PD-1 NPs remain to be elucidated. It is likely that the uptake of anti-PD-1 NPs by CD $11 c^{+}$DCs enhances the maturation and activation of DCs. We show enhanced maturation and activation by the increased expression of costimulatory receptors, including CD40, CD80, and CD86 in DCs. Once activated, the DCs can, in turn, activate effector T cells, thereby inducing an immune response against cancer cells. Our data demonstrate that the effective dosage of anti-PD-1 for evoking antitumor immunity in a murine melanoma cancer model could be reduced significantly by incorporating this therapeutic agent in NPs. This dose reduction was facilitated by a focused delivery of the NPs and their uptake by APCs in the SLTs. Our study demonstrates that treatment with anti-PD-1 NPs reduced tumor growth rate significantly in a murine melanoma model. Lastly, the anti-PD-1 mAb may promote the interaction of NPs with immune cells and internalization of the NPs. Such an interaction may increase the expression of adhesion molecules, enhancing the adhesive capacity of effector immune cells with target tumor cells.

In summary, we demonstrate the potential of an NP-based platform that delivers anti-PD-1 to the spleen to further enhance antitumor immunity in a murine melanoma model. The use of this platform significantly reduces the need for treatment with high dosage of anti-PD-1. Our data highlight the importance of SLTs in promoting antitumor immunity and in increasing the toxicity of anti-PD-1.

\section{Methods}

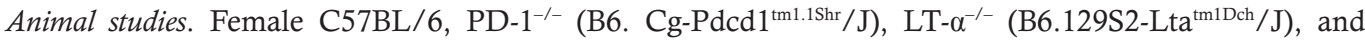
BALB/c mice (7-9 weeks old, body weight of 18-21 g) were purchased from The Jackson Laboratory.

Synthesis and characterization of NPs. PLGA-based NPs were prepared using a double-emulsion evaporation method. Briefly, 4.2 mg of mPEG-PLGA (Methoxy Poly[ethylene glycol]-b-Poly[D,L-lactide-co-glycolide]); Mw 5,000:30,000 Da; 50:50 LA:GA (w:w) copolymer (AK102, Polyscitech) was dissolved in ethyl acetate (Thermo Fisher Scientific) to form the oil phase. An aqueous solution of $70 \mu \mathrm{g}$ of anti-PD-1 mAb (Purified anti-mouse CD279 [PD-1] antibody [RMP1-14, BioLegend] or APC anti-mouse CD279 [PD-1] antibody [29F.1A12, BioLegend]) were added to the oil phase and emulsified by sonication for 60 seconds. The emulsified solution was added dropwise to $3 \mathrm{ml}$ of $1 \%$ aqueous polyvinyl alcohol solution (PVA, Mw $\sim 31,000$, MilliporeSigma) on high vortex and sonicated for 60 seconds. Then, the mixture was added to a $0.25 \%$ PVA aqueous solution $(20 \mathrm{ml})$ and stirred continuously at room temperature for 3 hours to permit solidification of the NPs and evaporation of ethyl acetate. The NPs were concentrated by centrifugation at 2,700 g, using Amicon Ultra-15 centrifugal filter units (MWCO $100 \mathrm{kDa}$, MilliporeSigma). Every $10 \mathrm{~min}$ utes, centrifugation was halted, and more NPs were added to the top of the filter unit. Following achievement of the desired concentration, the NPs were washed twice with Dulbecco's phosphate-buffered saline 
(DPBS) (Mediatech Inc.). A $0.5 \mathrm{ml}$ suspension of NPs was transferred to a new tube designated as the high dose. This suspension was diluted in half with DPBS to create the low-dose NPs.

Fluorescently labeled NPs were synthesized from mPEG-PLGA copolymer and IRDye 800CW Carboxylate (LI-COR) or CF660R, Maleimide (MilliporeSigma) by nanoprecipitation. Briefly, $5 \mathrm{mg}$ of the polymer was dissolved in acetone. Then, the dye was dissolved in methanol and added to the polymer solution. This solution $(1.0 \mathrm{ml})$ was added dropwise into a $0.015 \%$ PVA aqueous solution $(10 \mathrm{ml})$ under vigorous stirring to formulate IR800 NPs or CF660 NPs. The NP suspension was stirred uncovered for 3 hours at room temperature in a chemical hood to evaporate the organic solvent completely. Then, the NPs were concentrated and washed, as described above.

The size distribution of anti-PD-1 NPs was determined using Malvern Zetasizer Nano ZS. Measurements were made in triplicates at room temperature. The morphology of NPs was assessed with transmission electron microscopy (TEM, JEOL 1200EX).

In vitro release studies of anti-PD-1 $m A b$ from $N P s$. To study the release of anti-PD-1 mAb from NPs, the NPs were loaded onto the filter in an ultrafiltration centrifuge tube, and the bottom of the tube was filled with DPBS. Then, the samples were incubated in an orbital shaker (Forma; Thermo Fisher Scientific) at a speed of $200 \mathrm{rpm}$ at $37^{\circ} \mathrm{C}$. At predetermined intervals, $200 \mu 1$ of DPBS was removed from the bottom of the tube for analysis, and $200 \mu 1$ of fresh DPBS was added to maintain a constant volume. The concentration of released antibody was determined by ELISA. ELISA plates (96-Well Flat-Bottom Immuno Plate, MaxiSorp, Nonsterile PS, Thermo Fisher Scientific) were coated overnight with mouse rPD-1 (ACRO Biosystems, M5228), washed 3 times with DPBST, and blocked with $1 \mathrm{mg} / \mathrm{ml} \mathrm{BSA}$ in DPBST overnight, followed by 3 washes. Then, NP samples collected at the specified time points and anti-PD-1 mAb standard samples (with known concentration) were incubated in the precoated wells for 1 hour at $37^{\circ} \mathrm{C}$, followed by 4 washes with DPBS+Tween20 (DPBST). The plates were incubated with $0.25 \mu \mathrm{g} / \mathrm{ml}$ of HRP-conjugated goat anti-rat IgG antibody (MilliporeSigma, AP 136P) for 1 hour, washed 4 times with DPBST, and developed with the Tetramethylbenzidine Substrate Solution (TMB, eBioscience, 00-4201-52) as per manufacturer's instructions. Absorption at $450 \mathrm{~nm}$ was measured on a plate reader (VersaMAX, Molecular Devices). Empty NPs were used as the base, and the actual concentration of anti-PD-1 mAb was calculated by comparing the results to a standard curve.

$T$ cell proliferation assay and assessment of activation markers of DCs. The capacity of DCs to induce activation of $\mathrm{T}$ cells following treatment with anti-PD-1 NPs was assessed using an allogeneic MLR assay. Murine CD11 $\mathrm{c}^{+} \mathrm{DCs}$ (C57BL/6 mice) and $\mathrm{CD}^{+} \mathrm{T}$ cells (BALB/c mice) were isolated from spleens using magnetic beads conjugated with anti-mouse CD11c (Miltenyi Biotec) and the EasySep Mouse T Cell Isolation Kit (Stemcell Technologies), respectively.

CD11 ${ }^{+}$DCs (150,000 cells/well) were cultured in complete RPMI-1640 medium (Lonza), supplemented with 10\% FBS (Gemini Bio-Products), 1\% penicillin/streptomycin (Corning), and 1\% L-Glutamine (Corning) and were treated with free anti-PD-1 mAb (anti-PD-1), anti-PD-1 NPs, or empty NPs. After an incubation period of 24 hours, a portion of the CD $11 \mathrm{c}^{+}$DCs was analyzed for the presence of activation markers by flow cytometry. The remainder of the CD11 $\mathrm{c}^{+}$DCs was washed with DPBS, and allogeneic $\mathrm{CD}^{+} \mathrm{T}$ cells $(300,000$ cells/well) were added to these cells. The cells were incubated together for 48 hours at $37^{\circ} \mathrm{C}$ in a humidified atmosphere containing $5 \% \mathrm{CO}_{2}$. Then, the cultured cells were labeled with $\left[{ }^{3} \mathrm{H}\right]$-Thymidine $(0.25 \mu \mathrm{Ci}$ per well) for an additional 16 hours to measure $\mathrm{T}$ cell proliferation.

To study the potential role of DCs play in the release of anti-PD-1 mAb following the uptake of NPs, 150,000 DCs cells/well were cultured in complete RPMI-1640 medium and treated with APC-labeled anti-PD-1 NPs. After 1 hour of incubation, CD11 $\mathrm{c}^{+} \mathrm{DCs}$ were washed twice with DPBS, and CD3 ${ }^{+} \mathrm{T}$ cells (450,000 cells/well) were added to these cells. These cells were incubated together for 6 hours at $37^{\circ} \mathrm{C}$ in a humidified atmosphere containing $5 \% \mathrm{CO}_{2}$. Then, the $\mathrm{T}$ cells were examined by flow cytometric analysis.

Tumor implantation. B16-F10 murine melanoma cells were cultured in complete RPMI-1640 medium. Cells in exponential growth phase were harvested from culture, washed twice with sterile DPBS, counted, and resuspended in DPBS. For the prophylactic model, 200,000 murine B16-F10 melanoma cells were grafted s.c. into both flanks of C57BL/6 mice. For the therapeutic model, 25,000 B16-F10 murine melanoma cells were inoculated s.c. into the right flanks of C57BL/6 mice. Ten days after tumor cell inoculation, mice with tumors around $25-30 \mathrm{~mm}^{3}$ in size were randomized into different groups and given specified treatments: $100 \mu \mathrm{l}$ of anti-PD-1 NPs suspension $(1.8 \mu \mathrm{g}$ anti-PD-1 mAb loaded in $100 \mu \mathrm{l}$ of anti-PD-1 NPs), empty NPs, free anti-PD-1 (1.8 $\mu$ g anti-PD-1 mAb in DPBS), or vehicle (DPBS). From each group, 
3-4 mice were sacrificed 17 days following inoculation, and the tumors and spleens were collected for flow cytometric analyses. Antitumor activity was evaluated in terms of tumor volume (V), in which $l$ and $w$ are, respectively, the larger and smaller diameters of the tumor as measured by a digital caliper, defined as

$$
\left.V=\left(\frac{1}{6} \pi\right) \times l \times w^{2} \quad \text { (Equation } 1\right)
$$

The mean tumor growth inhibition (\%TGI), in which $\mathrm{V}_{\mathrm{c}}$ and $\mathrm{V}_{\mathrm{t}}$ are, respectively, the sizes of the control and treated groups at the end of the study (60) was calculated according to the formula:

$$
T G I(\%)=\left[1-\frac{\left(V_{t}\right)}{\left(V_{c}\right)}\right] \times 100 \quad \text { (Equation 2) }
$$

Tumor growth was assayed as described above until the experimental endpoint was reached or when excessive tumor burden or disease state required protocol-stipulated euthanasia.

In vivo trafficking of NPs. To study the trafficking of NPs, B16-F10 melanoma tumor-bearing mice $(n=3-4$ ) group) were anesthetized via inhalation of isoflurane/oxygen and IR800 NPs were administered i.v. Trafficking of IR800 NPs was studied using a UVP iBOX Explorer Imaging Microscope (UVP) equipped with a 750-780 $\mathrm{nm}$ excitation filter and an $800 \mathrm{~nm}$ long-pass emission filter. For live imaging, mice were anesthetized and placed inside the dark box in prone position. For ex vivo imaging, mice were sacrificed via $\mathrm{CO}_{2}$ inhalation and cervical dislocation. Tumors and spleens were harvested for imaging.

Splenectomy. C57BL/6 and LT- $\alpha^{-/-}$mice were anesthetized via inhalation of isoflurane/oxygen. Then, a $1-\mathrm{cm}$ incision was made in the skin on the left upper abdomen, and the spleen was removed. The splenic artery and vein were tied, and the abdominal wall was closed with a 6-0 silk running suture.

Flow cytometry. To prepare single-cell suspensions from spleens, spleen was minced into small fragments in DPBS and placed onto a 70- $\mu \mathrm{m}$ cell strainer (BD Falcon) attached to a 50-ml conical tube. Next, fragments of the spleen were pressed through a strainer using the plunger end of a syringe and washed through the strainer with excess DPBS. Next, the cell suspension was centrifuged at $550 \mathrm{~g}$ for 5 minutes. The supernatant was discarded, and the cell pellet was resuspended in $1 \mathrm{ml} \mathrm{ACK} \mathrm{lysis} \mathrm{buffer} \mathrm{(Lonza)} \mathrm{for} 2$ minutes. DPBS (30 ml) was added to dilute the lysis buffer, and the cell suspension was centrifuged again. After discarding the supernatant, the cell pellet was resuspended in RPMI-1640 to a concentration of $1 \times$ $10^{7}$ cells $/ \mathrm{ml}$. The same protocol was used for preparing single-cell suspensions from melanoma tumors.

Single-cell suspensions were plated in 96-well round-bottom plates (Corning) for intracellular cytokine staining and 96-well V-bottom plates (Corning) for surface and intracellular transcription factor staining. For intracellular IFN- $\gamma$ staining, the single-cell suspensions were stimulated with phorbol 12-mystirate 13-acetate (100 ng/ml, MilliporeSigma), ionomycin ( $1 \mu \mathrm{g} / \mathrm{ml}$, MilliporeSigma), and GolgiStop protein transport inhibitor (BD Biosciences) at $37^{\circ} \mathrm{C}$ for 4 hours prior to staining. Then, the cells were stained with Fixable Viability Dye (eBioscience Fixable Viability Dye eFluor450) diluted 1:1,000 $\mu 1$ in DPBS for 30 minutes at ${ }^{\circ} \mathrm{C}$. Next, the cells were washed with FACS buffer (DPBS + 2\% FBS + 1 mM EDTA + 0.1\% sodium azide) and incubated for 25 minutes at $4^{\circ} \mathrm{C}$ with fluorochrome-conjugated murine antibodies against CD3 (17A2), CD8a (53-6.7), CD4 (GK1.5), CD62L (MEL-14), CD44 (IM7), CD25 (PC61), CD69 (H1.2F3), PD-1 (RMP1-30), CD45 (30-F11), CD45R/B220 (RA3-6B2), F4/80 (BM8), CD11b (M1/70), CD11c (N418; eBioscience), CD40 (3/23), CD80 (16-10A1; eBioscience), and CD86 (GL1 [RUO]; BD Biosciences). Antibodies were purchased from BioLegend, unless otherwise stated. All cell-surface antibodies were diluted 1:300 $\mu$ in FACS buffer. The cells were permeabilized using the eBioscience Intracellular Fixation and Permeabilization Buffer Set (Thermo Fisher Scientific) for 30 minutes at $4^{\circ} \mathrm{C}$. Next, they were incubated with fluorochrome-labeled intracellular antibodies against IFN- $\gamma$ (XMG1.2) and FoxP3 (MF-14) diluted 1:300 $\mu 1$ in permeabilization buffer. Cells were washed once with permeabilization buffer and fixed in FACS buffer + $1 \%$ formalin. Flow cytometry was performed with a BD FACSCanto II flow cytometer (BD Biosciences). Analysis of flow cytometry data was performed by FlowJo (FlowJo).

Luminex assay for quantification of cytokines. After sacrificing mice, blood was obtained and centrifuged at 2,000 $\mathrm{g}$ for 10 minutes, and serum samples were collected. The concentration of cytokines was detected in these samples using the MILLIPLEX MAP Mouse Cytokine/Chemokine Magnetic 
Kit (MilliporeSigma), as per manufacturer's instructions. Median fluorescence intensity was measured by a Luminex 200 IS instrument and analyzed using the logistic curve-fitting method to determine cytokine concentrations.

Histology and immunofluorescence imaging. To study the cellular uptake of NPs in vitro, cultured splenocytes were incubated with fluorescently labeled NPs for 3 hours at $37^{\circ} \mathrm{C}$ and washed 3 times with DPBS. The samples were then stained with FITC-labeled anti-CD11b (M1/70; BioLegend), anti-CD11c (N418; eBioscience), and anti-F4/80 antibodies (BM8; BioLegend), as well as mounting medium with DAPI (VECTASHIELD, Vector Laboratories Burlingame).

For H\&E staining, tissues were fixed in $10 \%$ formalin solution and embedded in paraffin blocks. Sections were cut and stained with $\mathrm{H} \& \mathrm{E}$ by conventional techniques.

For immunofluorescence imaging, mice tissues were flash-frozen in OCT compound (Tissue-Tek, Thermo Fisher Scientific) and cut into $8-\mu \mathrm{m}$ sections with a cryomicrotome. Sections were fixed in cold acetone for 10 minutes and then blocked with 3\% BSA for 30 minutes at room temperature. Next, the sections were incubated overnight at $4^{\circ} \mathrm{C}$ with primary antibodies: anti-CD3 antibody (SP7, Abcam; or 17A2, eBioscience) and anti-insulin antibody (Abcam). The sections were then incubated with secondary antibodies: AlexaFluor 488 goat anti-rabbit IgG (catalog A27034, Thermo Fisher Scientific), AlexaFluor 594 goat anti-rat IgG (catalog A-11007, Thermo Fisher Scientific), and AlexaFluor 594 goat anti-rabbit IgG (catalog A-11037, Thermo Fisher Scientific) for 30 minutes at room temperature. FITC-conjugated anti-CD11b (M1/70; BioLegend) antibody was used for CD11b staining. Finally, the slides were washed with DPBS for 5 minutes, stained with DAPI, and coverslipped.

Data Availability. All data generated or analyzed during this study are available from the corresponding author on reasonable request.

Statistics. A 2-tailed Student's $t$ test or 1-way ANOVA was used to determine statistical significance between 2 groups and several groups, respectively. Differences in survival in each group were determined using the Kaplan-Meier method, and the overall comparisons of slopes were carried out using linear regression model (GraphPad Prism 5.0). Data represent means \pm SEM. ${ }^{*} P<0.05 ;{ }^{*} P<0.01 ;{ }^{* *} P<0.005$.

Study approval. All animal experiments were approved and performed in accordance with the guidelines and regulations of the IACUC of the Brigham and Women's Hospital and Harvard Medical School in Boston, Massachusetts, USA.

\section{Author contributions}

FO performed experiments, analyzed and interpreted data, drafted, critically revised, and finalized the manuscript. MU, VK, LD, SKE, BB, and MY performed experiments. JRA, YH, PTS, GFM, NA, TS, and IG critically revised the manuscript. RA designed the study, interpreted the data, critically revised, and finalized the manuscript.

\section{Acknowledgments}

This work is supported in part by the NPRP grant 9-350-3-074 (RA) and NIH/NCI grant 1R01 CA190838-01A1 (TS).

Address correspondence to: Reza Abdi, Transplant Research Center, Brigham and Women's Hospital, 221 Longwood Ave, Boston Massachusetts 02115, USA. Phone: 617.732.7249; Email: rabdi@rics.bwh.harvard.edu.

1. Sallusto F, Lanzavecchia A. The instructive role of dendritic cells on T-cell responses. Arthritis Res. 2002;4 Suppl 3:S127-S132.

2. Stockwin LH, McGonagle D, Martin IG, Blair GE. Dendritic cells: immunological sentinels with a central role in health and disease. Immunol Cell Biol. 2000;78(2):91-102.

3. Bandola-Simon J, Roche PA. Dysfunction of antigen processing presentation by dendritic cells in cancer [published online ahead of print April 5, 2018]. Mol Immunol. https://doi.org/10.1016/j.molimm.2018.03.025.

4. Boenisch O, Sayegh MH, Najafian N. Negative T-cell costimulatory pathways: their role in regulating alloimmune responses. Curr Opin Organ Transplant. 2008;13(4):373-378.

5. Schuchmann M, et al. The programmed death (PD)-1/PD-ligand 1 pathway regulates graft-versus-host-reactive CD8 T cells after liver transplantation. Am J Transplant. 2008;8(11):2434-2444.

6. Sharma P, Hu-Lieskovan S, Wargo JA, Ribas A. Primary, Adaptive, and Acquired Resistance to Cancer Immunotherapy. Cell. 2017;168(4):707-723.

7. Naidoo J, et al. Toxicities of the anti-PD-1 and anti-PD-L1 immune checkpoint antibodies. Ann Oncol. 2015;26(12):2375-2391. 
8. Weinstock M, McDermott D. Targeting PD-1/PD-L1 in the treatment of metastatic renal cell carcinoma. Ther Adv Urol. 2015;7(6):365-377.

9. Meng X, Huang Z, Teng F, Xing L, Yu J. Predictive biomarkers in PD-1/PD-L1 checkpoint blockade immunotherapy. Cancer Treat Rev. 2015;41(10):868-876.

10. Wolchok JD, et al. Overall Survival with Combined Nivolumab and Ipilimumab in Advanced Melanoma. N Engl J Med. 2017;377(14):1345-1356.

11. Milling L, Zhang Y, Irvine DJ. Delivering safer immunotherapies for cancer. Adv Drug Deliv Rev. 2017;114:79-101.

12. Cousin S, Seneschal J, Italiano A. Toxicity profiles of immunotherapy. Pharmacol Ther. 2018;181:91-100.

13. Zou W, Wolchok JD, Chen L. PD-L1 (B7-H1) and PD-1 pathway blockade for cancer therapy: Mechanisms, response biomarkers, and combinations. Sci Transl Med. 2016;8(328):328rv4.

14. Iwai Y, Hamanishi J, Chamoto K, Honjo T. Cancer immunotherapies targeting the PD-1 signaling pathway. J Biomed Sci. 2017;24(1):26

15. LaFleur MW, Muroyama Y, Drake CG, Sharpe AH. Inhibitors of the PD-1 Pathway in Tumor Therapy. J Immunol. 2018;200(2):375-383.

16. Li Y, et al. Hydrogel dual delivered celecoxib and anti-PD-1 synergistically improve antitumor immunity. Oncoimmunology. 2016;5(2):e1074374

17. Wang C, Ye Y, Hochu GM, Sadeghifar H, Gu Z. Enhanced Cancer Immunotherapy by Microneedle Patch-Assisted Delivery of Anti-PD1 Antibody. Nano Lett. 2016;16(4):2334-2340.

18. Li SY, et al. Restoring anti-tumor functions of T cells via nanoparticle-mediated immune checkpoint modulation. $J$ Control Release. 2016;231:17-28.

19. Kapadia CH, Perry JL, Tian S, Luft JC, DeSimone JM. Nanoparticulate immunotherapy for cancer. J Control Release. 2015;219:167-180.

20. Luo L, et al. Sustained release of anti-PD-1 peptide for perdurable immunotherapy together with photothermal ablation against primary and distant tumors. J Control Release. 2018;278:87-99.

21. Shao K, Singha S, Clemente-Casares X, Tsai S, Yang Y, Santamaria P. Nanoparticle-based immunotherapy for cancer. ACS Nano. 2015;9(1):16-30.

22. Schmid D, et al. T cell-targeting nanoparticles focus delivery of immunotherapy to improve antitumor immunity. Nat Commun 2017;8(1):1747.

23. Bronte V, Pittet MJ. The spleen in local and systemic regulation of immunity. Immunity. 2013;39(5):806-818.

24. Swirski FK, et al. Identification of splenic reservoir monocytes and their deployment to inflammatory sites. Science. 2009;325(5940):612-616.

25. Cortez-Retamozo V, et al. Origins of tumor-associated macrophages and neutrophils. Proc Natl Acad Sci USA. 2012;109(7):2491-2496.

26. Ugel S, et al. Immune tolerance to tumor antigens occurs in a specialized environment of the spleen. Cell Rep. 2012;2(3):628-639.

27. Dutta P, et al. Myocardial infarction accelerates atherosclerosis. Nature. 2012;487(7407):325-329.

28. Robbins CS, et al. Extramedullary hematopoiesis generates Ly-6C(high) monocytes that infiltrate atherosclerotic lesions. Circulation. 2012;125(2):364-374.

29. Cortez-Retamozo V, et al. Angiotensin II drives the production of tumor-promoting macrophages. Immunity. 2013;38(2):296-308

30. Banks TA, et al. Lymphotoxin-alpha-deficient mice. Effects on secondary lymphoid organ development and humoral immune responsiveness. J Immunol. 1995;155(4):1685-1693.

31. Koelzer VH, et al. Systemic inflammation in a melanoma patient treated with immune checkpoint inhibitors-an autopsy study. J Immunother Cancer. 2016;4:13.

32. Messenheimer DJ, et al. Timing of PD-1 Blockade Is Critical to Effective Combination Immunotherapy with Anti-OX40. Clin Cancer Res. 2017;23(20):6165-6177.

33. Arlauckas SP, et al. In vivo imaging reveals a tumor-associated macrophage-mediated resistance pathway in anti-PD-1 therapy. Sci Transl Med. 2017;9(389):eaal3604.

34. Michot JM, et al. Immune-related adverse events with immune checkpoint blockade: a comprehensive review. Eur J Cancer. 2016;54:139-148

35. Kamath AV. Translational pharmacokinetics and pharmacodynamics of monoclonal antibodies. Drug Discov Today Technol. 2016;21-22:75-83

36. Wang W, Wang EQ, Balthasar JP. Monoclonal antibody pharmacokinetics and pharmacodynamics. Clin Pharmacol Ther 2008;84(5):548-558

37. Dostalek M, Gardner I, Gurbaxani BM, Rose RH, Chetty M. Pharmacokinetics, pharmacodynamics and physiologically-based pharmacokinetic modelling of monoclonal antibodies. Clin Pharmacokinet. 2013;52(2):83-124.

38. Streilein JW. Ocular immune privilege: therapeutic opportunities from an experiment of nature. Nat Rev Immunol. 2003;3(11):879-889.

39. Schwarz RE, Hiserodt JC. Effects of splenectomy on the development of tumor-specific immunity. J Surg Res. 1990;48(5):448-453.

40. Wilhelm S, et al. Analysis of nanoparticle delivery to tumours. Nat Rev Mater. 2016;1:16014.

41. Leuschner F, et al. Therapeutic siRNA silencing in inflammatory monocytes in mice. Nat Biotechnol. 2011;29(11):1005-1010

42. Martin AJ, McCarthy D, Waltenbaugh C, Goings G, Luo X, Miller SD. Ethylenecarbodiimide-treated splenocytes carrying male CD4 epitopes confer histocompatibility Y chromosome antigen transplant protection by inhibiting CD154 upregulation. J Immunol. 2010;185(6):3326-3336.

43. Velpurisiva P, Gad A, Piel B, Jadia R, Rai P. Nanoparticle Design Strategies for Effective Cancer Immunotherapy. J Biomed (Syd). 2017;2(2):64-77

44. Fan Y, Moon JJ. Nanoparticle Drug Delivery Systems Designed to Improve Cancer Vaccines and Immunotherapy. Vaccines (Basel). 2015;3(3):662-685.

45. Irvine DJ, Hanson MC, Rakhra K, Tokatlian T. Synthetic Nanoparticles for Vaccines and Immunotherapy. Chem Rev. 2015;115(19):11109-11146.

46. Nishino M, Sholl LM, Hodi FS, Hatabu H, Ramaiya NH. Anti-PD-1-Related Pneumonitis during Cancer Immunotherapy. 
N Engl J Med. 2015;373(3):288-290.

47. Nishino M, et al. PD-1 Inhibitor-Related Pneumonitis in Advanced Cancer Patients: Radiographic Patterns and Clinical Course. Clin Cancer Res. 2016;22(24):6051-6060.

48. Wu J, Hong D, Zhang X, Lu X, Miao J. PD-1 inhibitors increase the incidence and risk of pneumonitis in cancer patients in a dose-independent manner: a meta-analysis. Sci Rep. 2017;7:44173.

49. Kleffel S, et al. Melanoma Cell-Intrinsic PD-1 Receptor Functions Promote Tumor Growth. Cell. 2015;162(6):1242-1256.

50. Overacre-Delgoffe AE, et al. Interferon- $\gamma$ Drives Treg Fragility to Promote Anti-tumor Immunity. Cell. 2017;169(6):1130-1141.e11.

51. Abiko K, et al. IFN- $\gamma$ from lymphocytes induces PD-L1 expression and promotes progression of ovarian cancer. Br J Cancer. 2015;112(9):1501-1509.

52. Zaidi MR, Merlino G. The two faces of interferon- $\gamma$ in cancer. Clin Cancer Res. 2011;17(19):6118-6124.

53. Dobrzanski MJ, Reome JB, Dutton RW. Role of effector cell-derived IL-4, IL-5, and perforin in early and late stages of type 2 CD8 effector cell-mediated tumor rejection. J Immunol. 2001;167(1):424-434.

54. Azzi J, et al. Targeted Delivery of Immunomodulators to Lymph Nodes. Cell Rep. 2016;15(6):1202-1213.

55. Preston CC, et al. The ratios of CD8+ T cells to CD4+CD25+ FOXP3+ and FOXP3- T cells correlate with poor clinical outcome in human serous ovarian cancer. PLoS One. 2013;8(11):e80063.

56. Alsaab HO, et al. PD-1 and PD-L1 Checkpoint Signaling Inhibition for Cancer Immunotherapy: Mechanism, Combinations, and Clinical Outcome. Front Pharmacol. 2017;8:561.

57. Melief CJ. Cancer immunotherapy by dendritic cells. Immunity. 2008;29(3):372-383.

58. Nouri-Shirazi M, et al. Dendritic cells capture killed tumor cells and present their antigens to elicit tumor-specific immune responses. J Immunol. 2000;165(7):3797-3803.

59. Spitzer MH, et al. Systemic Immunity Is Required for Effective Cancer Immunotherapy. Cell. 2017;168(3):487-502.e15.

60. Hather $\mathrm{G}$, et al. Growth rate analysis and efficient experimental design for tumor xenograft studies. Cancer Inform. 2014;13(Suppl 4):65-72. 